OPEN ACCESS

Edited by:

Nicola Fusco,

University of Milan, Italy

Reviewed by:

Donald Moore,

Levine Cancer Institute, United States Konstantinos Venetis,

University of Milan, Italy

${ }^{*}$ Correspondence:

Huihui L

huihuili82@163.com

Specialty section: This article was submitted to

Breast Cancer,

a section of the journal

Frontiers in Oncology

Received: 09 November 2021 Accepted: 25 January 2022

Published: 16 February 2022

Citation:

Chi Y, Shang M, Xu L, Gong H, Tao R,

Song L, Zhang B, Yin S, Cong B and $\mathrm{Li} H$ (2022) Durable Effect of Pyrotinib and Metronomic Vinorelbine in HER2-

Positive Breast Cancer With

Leptomeningeal Disease: A Case Report and Literature Review.

Front. Oncol. 12:811919. doi: 10.3389/fonc.2022.811919

\section{Durable Effect of Pyrotinib and Metronomic Vinorelbine in HER2- Positive Breast Cancer With Leptomeningeal Disease: A Case Report and Literature Review}

\author{
Yajing Chi ${ }^{1,2}$, Mao Shang ${ }^{3}$, Liang $\mathrm{Xu}^{4}$, Heyi Gong ${ }^{5}$, Rongjie Tao ${ }^{6}$, Lihua Song ${ }^{1}$, \\ Baoxuan Zhang ${ }^{1}$, Sha Yin ${ }^{1}$, Binbin Cong $^{7}$ and Huihui $L^{1{ }^{1 *}}$
}

\begin{abstract}
${ }^{1}$ Department of Breast Medical Oncology, Shandong Cancer Hospital and Institute, Shandong First Medical University and Shandong Academy of Medical Sciences, Jinan, China, ${ }^{2}$ School of Medicine, Nankai University, Tianjin, China, ${ }^{3}$ Department of Oncology, Jinan Central Hospital, Cheeloo College of Medicine, Shandong University, Jinan, China, ${ }^{4}$ Department of Radiology, Shandong Cancer Hospital and Institute, Shandong First Medical University and Shandong Academy of Medical Sciences, Jinan, China, ${ }^{5}$ Department of Radiation Oncology, Shandong Cancer Hospital and Institute, Shandong First Medical University and Shandong Academy of Medical Sciences, Jinan, China, ${ }^{6}$ Department of Neurosurgery, Shandong Cancer Hospital and Institute, Shandong First Medical University and Shandong Academy of Medical Sciences, Jinan, China, 7 Department of Breast Surgery, Shandong Cancer Hospital and Institute, Shandong First Medical University and Shandong Academy of Medical Sciences, Jinan, China
\end{abstract}

Leptomeningeal metastases (LM) are rare and catastrophic for metastatic breast cancer (MBC). The prognosis of HER2-positive breast cancer (BC) with $L M$ is extremely poor. There is no high-quality evidence of treatment regimens in HER2-positive BC with LM yet. Here, we present a case of LM in a 50-year-old woman with HER2-positive BC. Immunohistochemistry revealed invasive ductal carcinoma, estrogen receptor negative, progesterone receptor negative, HER2 3+, P53 positive 80\%, and Ki-67 positive 35\%. Reported for the first time, the patient was given pyrotinib-targeted therapy (400 mg, oral, every day), metronomic vinorelbine (40 mg, oral, three times a week), and intrathecal methotrexate (10 mg, infrequent and irregular use due to poor compliance) synchronously. The patient received and benefited from the treatment regimen for 16 months. And the quality of life, as self-reported, improved significantly. We also comprehensively summarized all the case reports, observational studies, and clinical trials related to HER2-positive BC with LM in the PubMed database and ClinicalTrials.gov. Intrathecal chemotherapy (methotrexate, cytarabine, thiotepa), intrathecal trastuzumab, whole-brain radiotherapy, and systemic therapy are commonly used treatment options according to a review of the literature and research. Pembrolizumab and trastuzumab deruxtecan (DS-8201) as novel drugs are promising in LM. Furthermore, trastuzumab emtansine (T-DM1) and tyrosine kinase inhibitors (TKIs) such as tucatinib and neratinib have exhibited good efficacy in HER2-positive BC with central nervous system (CNS) metastases and deserve further exploration. In our report, combining pyrotinib-targeted 
therapy with metronomic chemotherapy is a potential regimen, which has presented satisfactory therapeutic efficacy and also warrants additional investigation in HER2positive BC with LM.

Keywords: case report, leptomeningeal metastases, HER2-positive breast cancer, pyrotinib, metronomic vinorelbine

\section{INTRODUCTION}

Breast cancer $(\mathrm{BC})$ is the most widespread cancer and the primary tumor-related reason for death in women around the globe (1). Notably, accounting for $1 \%$ to $5 \%$ in BC, leptomeningeal metastases (LM) is closely linked to high mortality (2). The clinical symptoms of LM include headache, dizziness, nausea, vomiting, mental disorders, disturbances of consciousness, ataxia or weakness, etc. BC patients with LM have worse prognoses, compared to patients with metastases to other sites (3-5). The survival of untreated BC patients with LM is only about 1 month, and even after treatment, the average survival time seldom reach 8 months (6). Treatment choices mainly include radiotherapy, intrathecal chemotherapy, systemic treatment, and symptomatic care. There are still no available unified treatment protocols to date. Many treatment options exist, but combining or choosing among them remains difficult due to the limitations and of these therapies and their varying degree of tolerability by patients. Whether cerebrospinal radiotherapy can improve survival is still not clear enough. Intrathecal chemotherapy often exhibits potentially severe neurotoxicity, usually late in the course of treatment. In addition, systemic chemotherapy or targeted therapy has a limited ability to penetrate the blood-brain barrier.

Of all the subtypes, Human epidermal growth factor receptor 2 (HER2)-positive BC has a predilection for brain metastasis but is less likely to develop LM at the same time (7). Previous studies have reported that the frequency of HER2-positive $\mathrm{BC}$ among LM patients ranges between 14\%-29\% (8-12). Anti-HER2 targeted therapy is the preferred treatment regimen for patients with HER2-positive BC, and those with LM are no exception to this rule. Patients with LM, on the other hand, tend to have higher Eastern Cooperative Oncology Group (ECOG) scores $(>2)$, which might make traditional systemic treatments like chemotherapy and cerebrospinal radiotherapy difficult. There is also inadequate evidence to demonstrate the effectiveness of traditional macromolecular anti-HER2 drugs such as trastuzumab through the blood-brain barrier.

How to solve the aforementioned issues and enable patients to achieve long-term survival has a long way to go. Currently, most of the relevant research and clinical practice are still in their infancy. Herein, we present a case of HER2-positive BC with LM who was mainly treated with a combination of pyrotinibtargeted therapy and metronomic vinorelbine, resulting in a sustained therapeutic benefit for up to 16 months.

\section{CASE REPORT}

A 50-year old woman with no noteworthy medical history was diagnosed with left $\mathrm{BC}$ a decade ago and underwent a modified radical mastectomy: the left breast harbored an invasive ductal carcinoma. Additionally, 5 of 5 left intermuscular lymph nodes and 2 of 4 axillary lymph nodes were involved in metastasis. Immunohistochemistry found the presence of estrogen receptor negative, progesterone receptor negative, HER2 3+, P53 positive $80 \%$, and Ki-67 positive $35 \%$. Therewith, adjuvant chemotherapy consisting of 6 cycles of cyclophosphamide, epirubicin, and 5fluorouracil was given to the patient, as well as radiotherapy to the left chest wall and supraclavicular area (50 Gy/25 fractions).

In November 2016, the patient developed metastasis to the left 10th rib, sternum, multiple lymph node metastases in the retroperitoneum, abdominal cavity, and mediastinum, and complicated with pleural effusion. However, the patient only received 6 cycles of docetaxel plus cyclophosphamide at the local hospital. After first-line treatment, the lesions could not be measured. And there was no evidence of progression until September 2017, when the patient had a single metastatic lesion of the right cerebellum. Over the following days, the patient got intensity-modulated radiotherapy (50Gy/10 fractions) targeting cerebellar metastasis, along with 4 cycles of gemcitabine plus capecitabine and trastuzumab simultaneously at the local hospital. The patient continued receiving trastuzumab for a year and was subsequently re-examined regularly with no signs of recurrence or new metastasis.

In December 2018, the patient had progressive onset of headache, nausea, vomiting, lower back pain, urinary retention, abnormal sensation in both lower extremities, constipation, and anal distension. The patient was subsequently admitted to our hospital in February 2019 for further treatment. A somewhat enlarged metastasis lesion to the right cerebellum was discovered on magnetic resonance imaging (MRI) after admission (Figure 1A). There were also abnormal strengthening signals in bilateral ventricular ependyma, meninges, spinal meninges, and cauda equina regions, which were identified and considered as LM (Figures 1B, C). A neurosurgeon performed right ventriculoperitoneal shunt and left ventricular Omaya reservoir implantation on the patient, followed by intrathecal injection of methotrexate $(10 \mathrm{mg})$. A physician synchronously gave the patient pyrotinib-targeted therapy (400 mg, oral, daily), metronomic vinorelbine (40 mg, oral, three times a week), and intrathecal methotrexate (10 $\mathrm{mg}$, occasional and irregular use due to poor compliance) since February 27, 2019. All symptoms were significantly relieved after 2 cycles of treatment, and the patient was able to urinate on her own, get out of bed, and walk with the assistance of her family members, and the quality of life improved drastically. Per the Common Terminology Criteria for Adverse Events (CTCAE) version 5.0, the patient developed Grade 1 diarrhea during treatment. Meanwhile, the synchronous MRI showed that the cerebella lesion had 


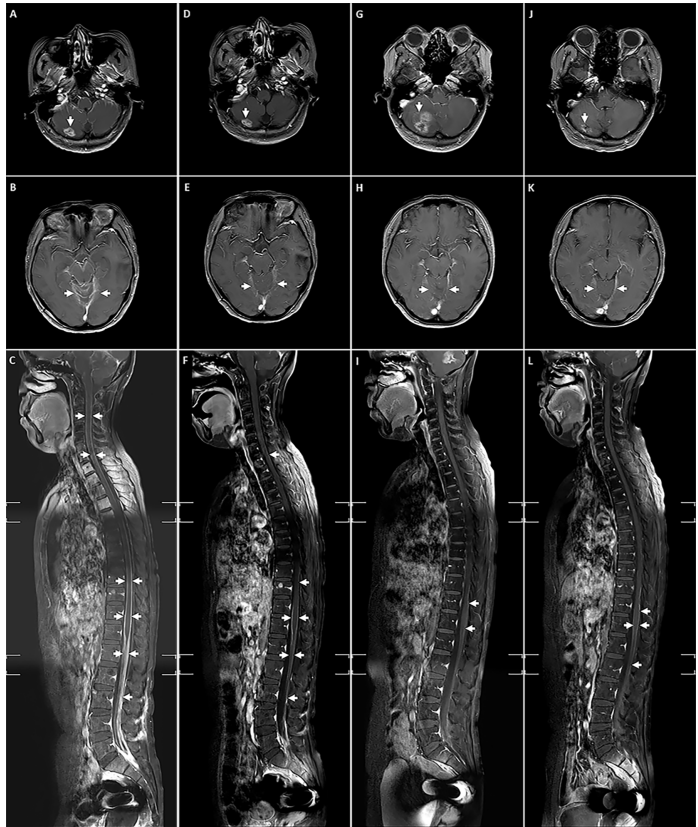

FIGURE 1 | The lesions of right cerebellum metastasis, LM and spinal LM during treatment. (A-C) Before receiving pyrotinib combined with chemotherapy; (D-F) After 2 cycles of pyrotinib combining with chemotherapy; (G-I) Before the addition of WBRT; (J-L) After the addition of WBRT.

stabilized and that LM shrunk significantly. The overall evaluation of the curative effect pointed to partial response according to Response Evaluation Criteria in Solid Tumors (RECIST) version 1.1 (Figures 1D-F). In February 2020, a new metastasis appeared in the right occipital lobe, and the lesion in the right cerebellum was larger than before (Figures 1G, 2A) but no LM progression (Figures 1H, I), so the patient received whole-brain radiotherapy (WBRT) (right occipital lobe lesion: 45Gy/10 fractions, whole-brain: 30Gy/10 fractions; considering that this was secondary radiotherapy to the brain, clinical target volume deducted the previous radiotherapy range of patient in 2017). After radiotherapy, the brain metastases shrunk significantly (Figures 1J, 2B), and the patient continues to benefit from pyrotinib and metronomic vinorelbine. Notably, LM has always been in remission (Figures $\mathbf{1 K}, \mathbf{L}$ ). So far, the patient's extracranial lesions have no signs of recurrence or metastasis, the central nervous system (CNS) metastases symptoms also have improved significantly beyond anyone's expectations, and she has benefited from the current treatment regimen for 16 months.

\section{METHODS}

Literature and research were searched comprehensively in PubMed database and ClinicalTrials.gov by using the following keywords: leptomeningeal metastasis, leptomeningeal carcinomatosis, meningeal metastasis, meningeal neoplasms, meningeal carcinomatosis, neoplastic meningitis, carcinomatous meningitis, breast cancer, HER2-positive, case report, case series, observational study, prospective study, retrospective study, intervention study, clinical trial. Then, by combing the search results, we summarized the case reports of HER2-positive BC with LM, observational studies, and clinical trials related to $\mathrm{LM}$ in $\mathrm{BC}$.

\section{RESULTS}

Twenty case reports, 19 of 28 observational studies, 9 of 10 ongoing clinical trials, and 4 of 18 completed clinical trials in our review related to $\mathrm{LM}$ in $\mathrm{BC}$ included HER2-positive $\mathrm{BC}$ patients (Figure 3). Among these, all case reports, 4 ongoing clinical trials, and 2 completed clinical trials were specialized in HER2positive BC with LM. Moreover, after an overview of 18 landmark clinical trials of HER2-positive MBC, only one clinical trial concerned LM. Literature review were summarized at following five tables.

\section{Case Reports of HER2-Positive BC With LM}

A total of 25 cases of HER2-positive BC with LM were included in the review [Table 1 (13-32)]. The median age of all patients was 43 years (range 31-75), with 9 cases of hormone receptor positive (47.3\%), 12 cases of invasive ductal carcinoma (70.5\%), and 19 cases $(76.0 \%)$ with CNS parenchyma metastases. Median progression-free survival (mPFS) for all patients was 16.8 months. In terms of treatment, fourteen patients were treated with intrathecal chemotherapy (methotrexate 92.8\%, cytarabine $28.5 \%$, thiotepa $14.2 \%$ ), about half of patients received intrathecal trastuzumab, and 9 patients received both. Any form of anti-HER2-targeted therapy, radiotherapy, and chemotherapy was given to 19,12 , and 21 patients, respectively.

\section{Observational Studies in BC With LM}

Finally, 28 observational studies were reviewed based on the search criteria [Table $2(9-11,33-57)$ ], with patients treated for LM ranging in time from 1976 to 2018. There was no report specifically addressed the LM of HER2-positive BC. However, a subset of HER2-positive patients was included in 18 of 28 studies. Intrathecal chemotherapy (methotrexate, cytarabine, and thiotepa, etc), WBRT, and systemic therapy were the main treatment options in all studies, with some patients receiving surgical intervention. The efficacy of combination therapy was assessed in 26 studies, with median progression-free survival (mPFS) ranging from 1.6 to 4.2 months for all patients, median overall survival (mOS) ranging from 0.9 to 7.7 months for all patients, and 1.5 to 8.4 months for HER2-positive patients. Only one retrospective study observed the efficacy of WBRT with a mOS of 2.1 months for all patients and 5.3 months for HER2positive patients. There was also only one case series that reviewed the efficacy of intravenous thiotepa alone in LM of $\mathrm{BC}$, with mOS rate of $69 \%$ and $31 \%$, respectively, at 6 months and 12 months. 

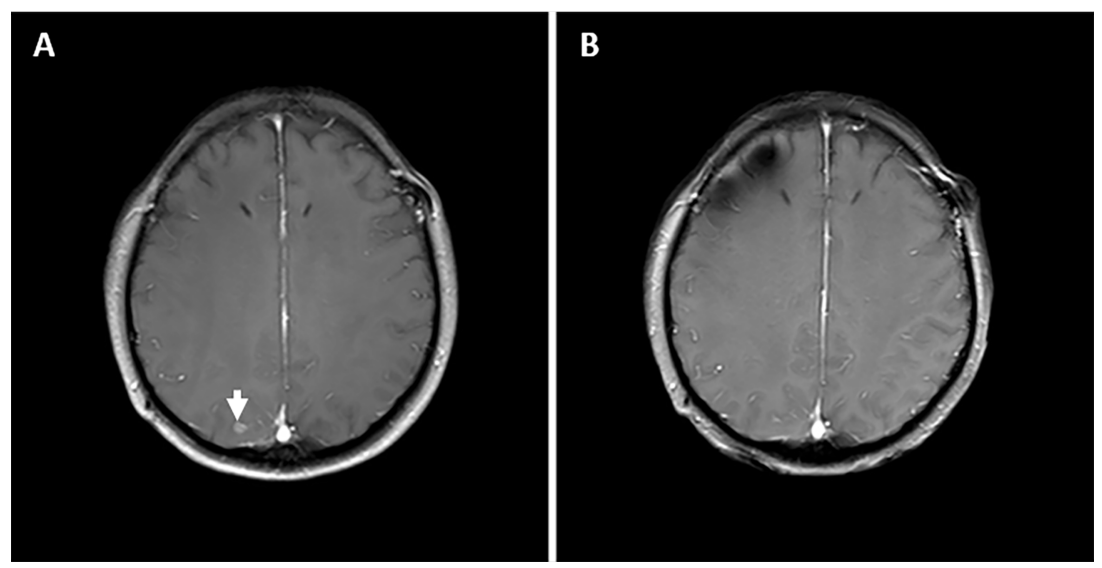

FIGURE 2 | The status of right occipital lobe metastasis lesion during treatment. (A) Before the addition of WBRT. (B) After the addition of WBRT.

\section{Clinical Trials in Solid Tumors With LM Including BC}

As far as we searched on ClinicalTrials. gov, until now, there were 10 ongoing clinical trials related to LM in BC [Table 3 (5867)]. Except for the ANGLeD study, the others were single-arm and phase I/II trials focused on HER2-positive/low expressing BC with LM. Novel anti-HER2-targeted drugs such as trastuzumab deruxtecan (DS-8201) and tucatinib are also being investigated. Currently, most of the clinical trials involving BC with LM that have obtained advances are designed as basket trials [Table 4 (68-85)].

In Table 4, 16 of 18 studies explored the efficacy of intrathecal treatment in LM, with mOS ranging from 2.0 to 7.3 months. The mOS of HER2-positive patients who received intrathecal trastuzumab could also reach 7.3 months in a phase I clinical trial. Two randomized controlled trials compared intrathecal chemotherapy with non-intrathecal chemotherapy in BC: a phase III study showed that intrathecal chemotherapy provided better survival compared to the control group (mPFS 3.8 vs. 2.2 months, $p=.04$; mOS 7.3 vs. 4.0 months, $p=.51$ ), but worse survival (mOS 4.58 vs. 7.58 months, $p=.32$ ) with treatmentrelated neurotoxicity in another study. According to a study in 1999, intrathecal cytarabine enhanced median intracranial PFS (1.93 vs. 1.0 months, $p=.007$ ) in comparison to methotrexate. Intravenous pembrolizumab achieved an objective response rate (ORR) of $61.5 \%$ in a recent phase II clinical trial of only 13 patients with LM in solid tumors (including BC).

\section{Landmark Clinical Trials of HER2- Positive MBC}

Eighteen landmark clinical trials for HER2-positive MBC were included in the literature review [Table 5 (86-110)]. Seven studies excluded patients with LM, and 10 studies did not clarify. Only the TBCRC 022 study allowed the inclusion of patients with LM while investigating the efficacy of neratinib plus capecitabine in HER2-positive BC with brain metastases (BM), and three patients with LM had progressive disease, partial response, and stable disease, respectively. The CLEOPATRA,

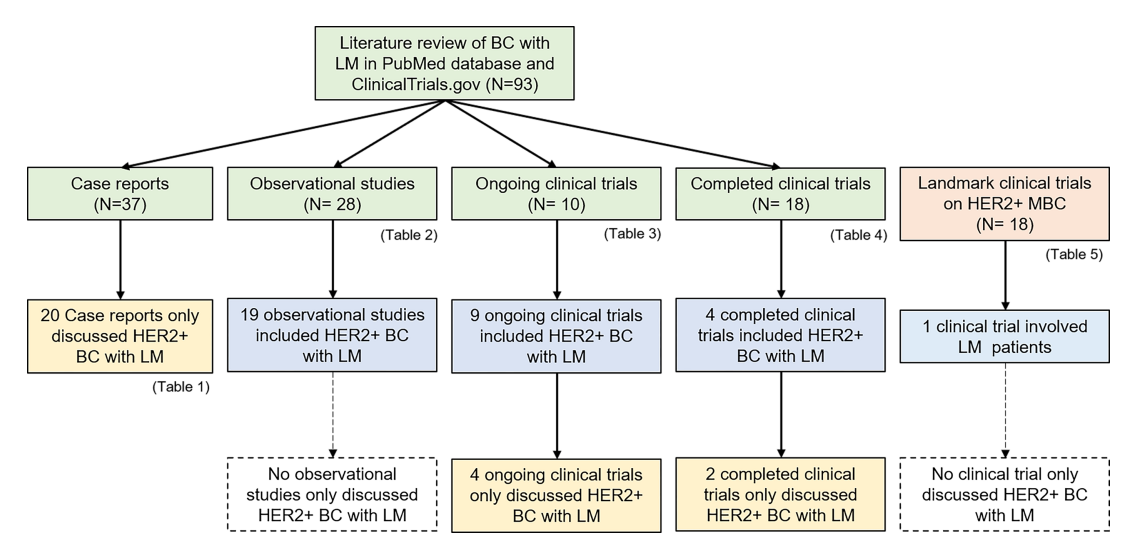

FIGURE 3 | The summary of literature review for HER2-positive BC with LM. 
TABLE 1 | SUmmary of HER2-positive breast cancer with leptomeningeal metastases in case reports.

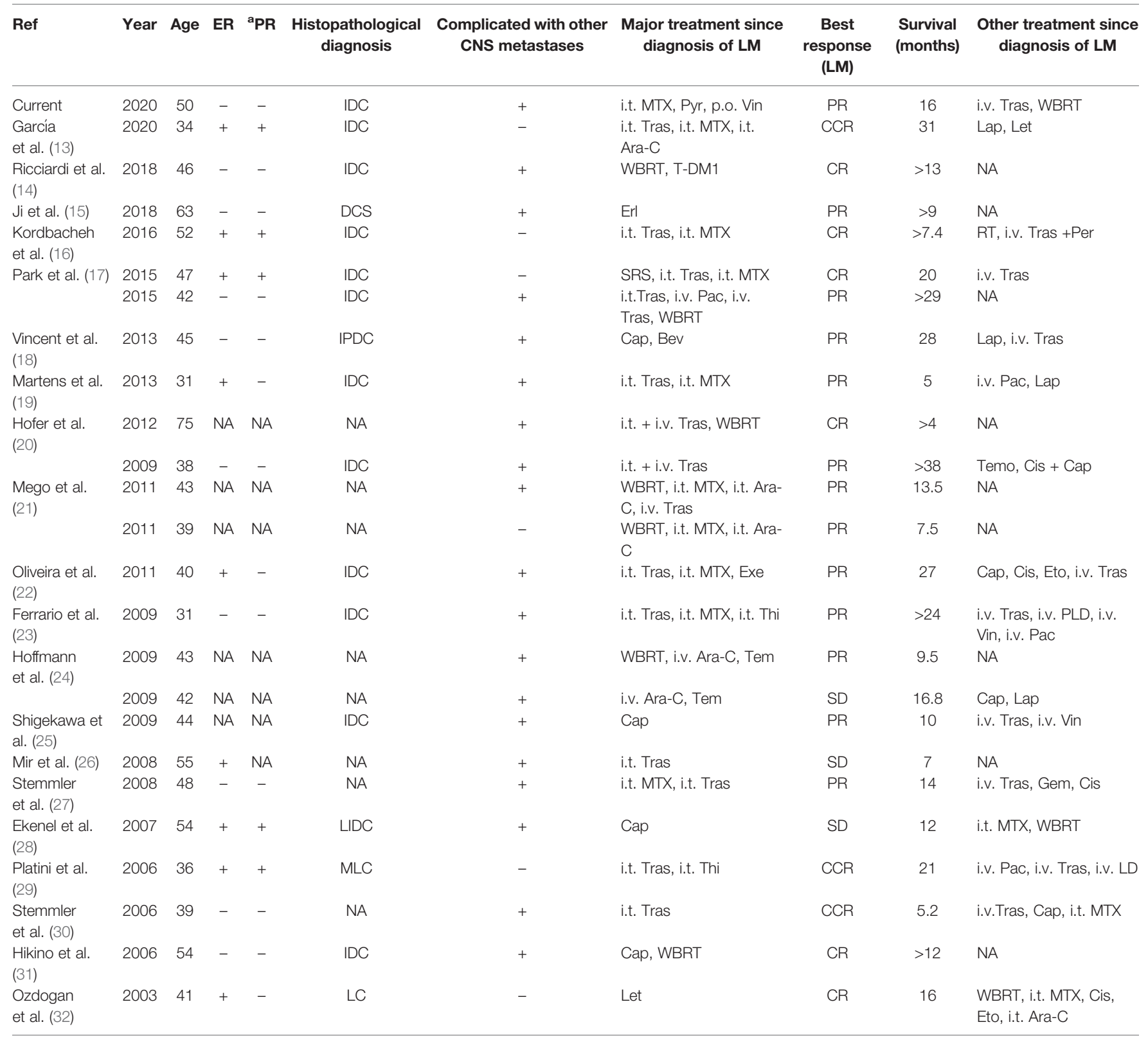

Ara-C, cytarabine; Bev, bevacizumab; Cap, capecitabine; CCR, CSF and clinical response; Cis, cisplatin; CR, complete remission; DCS, Ductal carcinoma in situ; ER, estrogen receptor; Erl, Erlotinib; Eto, etoposide; Exe, exemestane; Gem, gemcitabine; IDC, Invasive ductal carcinoma; IPDC, Invasive poorly differentiated carcinoma; i.t., intrathecal; i.v., intravenous; Lap, lapatinib; LC, lobular carcinoma; LD, liposomal doxorubicin; LIDC, lobular and infiltrative ductal carcinoma; Let, letrozole; MLC, Multifocal lobular carcinoma; MTX, methotrexate; NA, not available; Pac, paclitaxel; Per, pertuzumab; PLD, pegylated liposomal doxorubicin; p.o., per os; ${ }^{a} P R$, progesterone receptor; PR, partial remission; Pyr, pyrotinib; Ref, reference; RT, radiotherapy; SD, stable disease; SRS, Stereotactic radiotherapy; T-DM1, trastuzumab emtansine; Tem, temozolomide; Thi, thiotepa; Tras, trastuzumab; Vin, vinorelbine; WBRT, whole brain radiotherapy.

PUFFIN, ALTERNATIVE, and PHOEBE study completely excluded CNS metastases, whereas the CLEOPATRA study analyzed the occurrence of CNS metastases: the pertuzumab group took longer time than the placebo group to the onset CNS metastases (15.0 vs. 11.9 months, $p=.0049)$, and for patients with CNS metastases, the pertuzumab group seemed to have a longer survival (34.4 vs. 26.3 months, $p=.1139$ ). Although the BOLERO-3, PERUSE, and SOPHIA study included CNS metastases, no results have been reported for CNS metastases. In the DESTINY-Breast 01 study, DS-8201 reached a mPFS of 18.1 months in patients with BM. The mOS of patients with CNS metastasis treated by trastuzumab emtansine (T-DM1) in the EMILIA and TH3RESA study was 26.8 and 17.3 months, respectively, which was better than those of the control group. The LANDSCAPE study showed that the mPFS of patients with HER2-positive BM treated with lapatinib plus capecitabine was 
TABLE 2 | Summary of breast cancer with leptomeningeal metastases in observational studies.

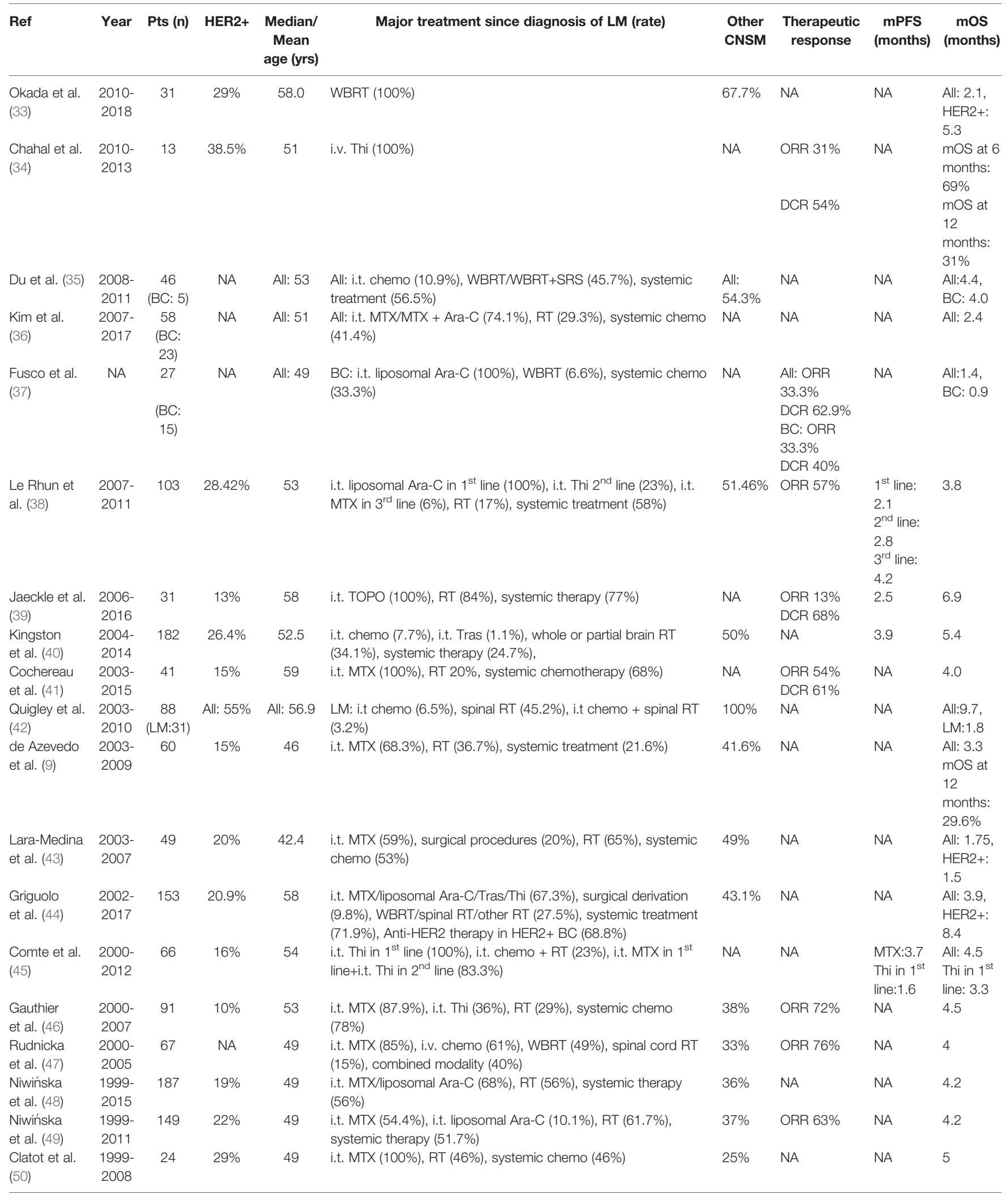


TABLE 2 | Continued

\begin{tabular}{|c|c|c|c|c|c|c|c|c|c|}
\hline Ref & Year & Pts (n) & HER2+ & $\begin{array}{l}\text { Median/ } \\
\text { Mean } \\
\text { age (yrs) }\end{array}$ & Major treatment since diagnosis of LM (rate) & $\begin{array}{l}\text { Other } \\
\text { CNSM }\end{array}$ & $\begin{array}{l}\text { Therapeutic } \\
\text { response }\end{array}$ & $\begin{array}{c}\text { mPFS } \\
\text { (months) }\end{array}$ & $\begin{array}{c}\text { mos } \\
\text { (months) }\end{array}$ \\
\hline $\begin{array}{l}\text { Morikawa } \\
\text { et al. (10) }\end{array}$ & $\begin{array}{l}1998- \\
2013\end{array}$ & 318 & $26 \%$ & 54 & i.t./i.v. therapy (14\%), RT (64\%), i.v. MTX (20\%), VP shunt (19\%) & $66 \%$ & NA & NA & $\begin{array}{l}\text { All: } 3.5 \text {, } \\
\text { HER2+: } \\
5.2\end{array}$ \\
\hline $\begin{array}{l}\text { Kosmas } \\
\text { et al. (51) }\end{array}$ & $\begin{array}{l}1996- \\
2000\end{array}$ & $\begin{array}{l}310 \\
\text { (LM: 7) }\end{array}$ & LM:28\% & LM: 54 & $\begin{array}{l}\text { LM: i.t. MTX+WBRT (71.4\%), i.t. MTX+RT to lumbar spine } \\
(14.3 \%)\end{array}$ & NA & $\begin{array}{l}\text { All: ORR } \\
28.6 \% \\
\text { DCR } 57.2 \% \\
\text { LM: ORR } \\
28.6 \%\end{array}$ & NA & LM: 3.6 \\
\hline $\begin{array}{l}\text { Yust-Katz } \\
\text { et al. (52) }\end{array}$ & $\begin{array}{l}1995- \\
2011\end{array}$ & 103 & $47.4 \%$ & 49.2 & $\begin{array}{l}\text { i.t. chemo (MTX 17\%, TOPO 22\%, Ara-C 24\%, Multiple 9\%), } \\
\text { WBRT (52.5\%), spinal RT (19\%), systemic chemo (36.4\%) }\end{array}$ & $46.1 \%$ & NA & NA & 4.2 \\
\hline $\begin{array}{l}\text { Lee et al. } \\
(11)\end{array}$ & $\begin{array}{l}1995- \\
2008\end{array}$ & 68 & $27.9 \%$ & $\begin{array}{l}\text { All: } 46 \\
\text { HER2 } \\
+: 47\end{array}$ & $\begin{array}{l}\text { All: i.t. chemo (14.7\%), WBRT (14.7\%), combination modality } \\
(61.8 \%) \\
\text { HER2+ (n=18): i.t. chemo (11.1\%), WBRT (11.1\%), combination } \\
\text { modality }(77.8 \%)\end{array}$ & $100 \%$ & NA & NA & $\begin{array}{l}\text { All: } 4.1 \\
\text { HER2+: } \\
5.9\end{array}$ \\
\hline $\begin{array}{l}\text { Chamberlain } \\
\text { et al. (53) }\end{array}$ & $\begin{array}{l}1990- \\
2007\end{array}$ & $\begin{array}{l}60 \\
(\mathrm{BC}: \\
20)\end{array}$ & NA & BC: 59.5 & BC: i.t. chemo (100\%), RT (90\%), systemic chemo (60\%) & NA & NA & NA & $\begin{array}{l}\text { All: } 1.5 \\
\text { BC: } 3\end{array}$ \\
\hline Yu et al. (54) & $\begin{array}{l}1990- \\
1999\end{array}$ & 8 & NA & 51.5 & i.t. MTX and/or immunotherapy (37.5\%), WBRT (100\%) & NA & NA & NA & $\begin{array}{l}\text { WBRT } \\
\text { alone: } 4.0 \\
\text { WBRT + } \\
\text { i.t. } \\
\text { therapy: } \\
5.37\end{array}$ \\
\hline $\begin{array}{l}\text { Chamberlain } \\
\text { et al. (55) }\end{array}$ & $\begin{array}{c}1986- \\
1995\end{array}$ & 32 & NA & 49 & $\begin{array}{l}\text { i.t. MTX in } 1^{\text {st }} \text { line }(65.3 \%) \text {, i.t. Ara-C } 2^{\text {nd }} \text { line }(44.9 \%) \text {, i.t. Thi in } \\
3^{\text {rd }} \text { line }(22.4 \%) \text {, RT }(42.9 \%)\end{array}$ & NA & $\begin{array}{l}\text { ORR: MTX } \\
43.8 \% \\
\text { Ara-C } 36.4 \% \\
\text { Thi } 27.3 \%\end{array}$ & NA & 7.5 \\
\hline $\begin{array}{l}\text { Jayson et al. } \\
(56)\end{array}$ & $\begin{array}{l}1979- \\
1992\end{array}$ & 35 & NA & 45 & $\begin{array}{l}\text { i.t. } \operatorname{MTX}(31.4 \%) \text {, i.t. Thi }(2.9 \%) \text {, i.t. Ara-C }(2.9 \%) \text {, } \\
\text { i.t. } \operatorname{MTX}+\mathrm{RT}(8.6 \%) \text {, RT (17.1\%) } \\
\text { i.v. } \operatorname{MTX}(28.6 \%) \text {, other systemic chemo }(14.3 \%)\end{array}$ & NA & DCR 63\% & NA & 2.57 \\
\hline $\begin{array}{l}\text { Strady et al. } \\
(57)\end{array}$ & $\begin{array}{l}1976- \\
1996\end{array}$ & $\begin{array}{l}41 \\
\text { (BC: } \\
18)\end{array}$ & NA & $\begin{array}{l}\text { All: } 57 \\
\text { BC: } 56\end{array}$ & BC: i.t. MTX (66.7\%), systemic chemo (77.8\%) & $\begin{array}{l}\text { All: } \\
39 \% \\
\text { BC: } \\
0 \%\end{array}$ & NA & NA & BC: 1.1 \\
\hline
\end{tabular}

Ara-C, cytarabine; BC, breast cancer; CNSM, central nervous system metastases; DCR, disease control rate; i.t., intrathecal; i.v., intravenous; LM, leptomeningeal metastases; MTX,

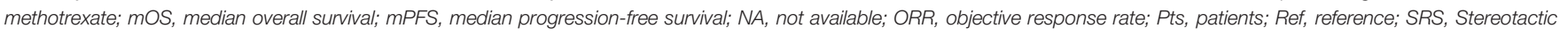
radiotherapy; $R T$, radiotherapy; Tras, trastuzumab; Thi, thiotepa; TOPO, topotecan; VP, ventriculoperitoneal; WBRT, whole brain radiotherapy.

5.5 months with an ORR of $65.9 \%$. In the BM subgroup of the HER2CLIMB study, the mPFS of tucatinib group was longer than the placebo group (7.6 vs. 5.4 months, $p<.001)$. In the NEfERT-T study, the neratinib group had a lower incidence of CNS recurrences than the trastuzumab group (8.3\% vs. $17.3 \%$, $p=.002)$, and also a lower overall cumulative incidence of intervention for CNS disease than the lapatinib group $(22.8 \%$ vs. $29.2 \%, p=.043)$ in NALA study.

\section{DISCUSSION}

Treatment for patients with LM should be based on multidisciplinary cooperation. According to the EANO-ESMO Clinical Practice Guidelines, patients with LM can choose intrathecal chemotherapy, radiotherapy, and systemic chemotherapy as the primary treatment (111). The above treatment regimens were also the predominant therapeutic options in the case reports and observational studies we reviewed (Tables 1, 2). However, there was rare large and randomized controlled clinical trial yet to demonstrate that the above therapeutic choices are efficient for BC with LM. Only two randomized controlled trials compared intrathecal chemotherapy with non-intrathecal chemotherapy in $\mathrm{BC}$ but came to opposite results. Furthermore, the majority of clinical trials only explored the efficacy of intrathecal treatment of LM with merely 7.3 months of optimal mOS (Table 4). In reviewed literature, $48 \%$ of the case reports and 26 of 28 observational studies of patients received radiotherapy, and most of the patients received systemic treatment. These studies, to various degrees, mirrored the effectiveness of radiotherapy and systemic treatment in patients with LM. Taking into account the poor physical condition of the current case, the radiologists reluctantly gave up the cerebrospinal radiotherapy that had been planned for her.

Anti-HER2 monoclonal antibody, antibody-drug conjugates, or tyrosine kinase inhibitors (TKIs) can be selected as targeted therapy regimens in HER2-positive MBC with LM. We noted 
TABLE 3 | Summary of ongoing clinical trials of breast cancer with leptomeningeal metastases registered on clinicaltrials.gov.

\begin{tabular}{|c|c|c|c|c|}
\hline NCT No./Study & Phase & Molecular status & Pts (n) & Study design \\
\hline NCT03501979/TBCRC049 (58) & $\|$ & HER2+ & 30 & Tuc + i.v. Tras + Cap \\
\hline NCT04588545 (59) & $|/| \mid$ & HER2+ & 39 & RT followed by i.t. Tras + i.t. Per \\
\hline NCT04420598/DEBBRAH (60) & $\|$ & HER2+/low expressing status & 39 & i.v. DS-8201 \\
\hline 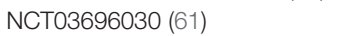 & 1 & HER2+ & 39 & i.v. HER2-Targeted Chimeric Antigen Receptor (HER2-CAR) T Cells \\
\hline NCT02650752 (62) & 1 & HER2+ & 11 & Lap + Cap \\
\hline NCT03613181/ANGLeD (63) & III & HER2- & 150 & ANG1005 vs. Physician's Best Choice \\
\hline 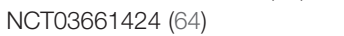 & 1 & Any & 16 & i.v. HER2 Bi-armed activated T-cells (BATs) \\
\hline NCT02422641 (65) & $\|$ & Any & 16 & i.v. high-dose MTX \\
\hline NCT01818713 (66) & $\|$ & Any & 6 & Glutathione pegylated liposomal doxorubicin hydrochloride formulation (2B3-101) \\
\hline NCT00992602 (67) & $\|$ & Any & 3 & i.v. high-dose MTX + i.t. liposomal Ara-C \\
\hline
\end{tabular}

Ara-C, cytarabine; Cap, capecitabine; DS-8201, trastuzumab deruxtecan; i.t., intrathecal; i.v., intravenous; Lap, lapatinib; MTX, methotrexate; Pts, patients; Per, pertuzumab; Ref, reference; $R T$, radiotherapy; Tras, trastuzumab; Tuc, tucatinib.

TABLE 4 | Summary of clinical trials in solid tumors (including breast cancer) with leptomeningeal metastases.

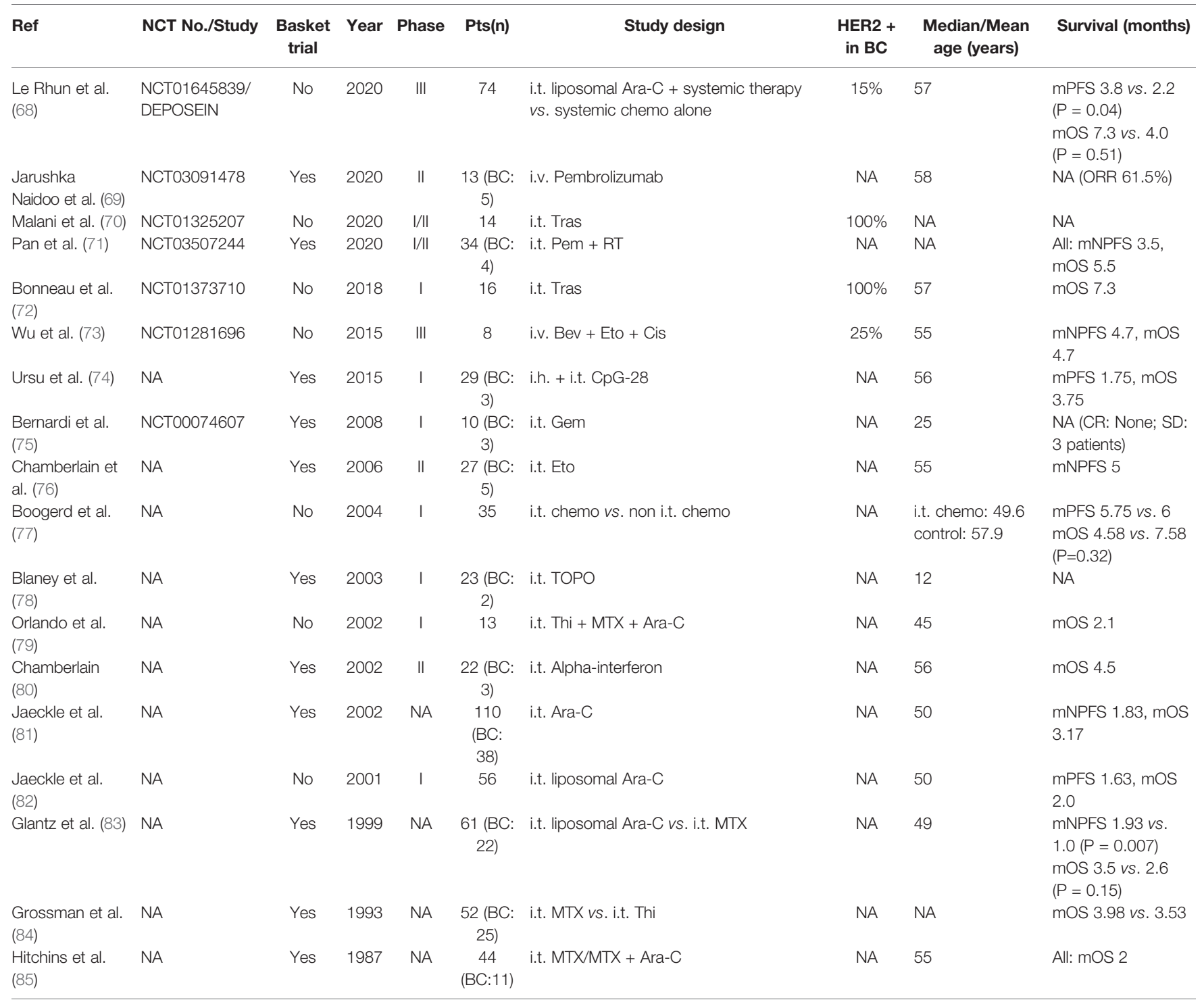

Ara-C, cytarabine; BC, breast cancer; Bev, bevacizumab; Cis, cisplatin; CLC, colon cancer; CNS, central nervous system; CR, complete response; Eto, etoposide; Gem, gemcitabine; i.h., hypodermic injection; i.t., intrathecal; i.v., intravenous; LC, lung cancer; MTX, methotrexate; mOS, median overall survival; mPFS, median progression-free survival; mNPFS, median neurologic progression-free survival; NA, not available; NSCLC, non-small cell lung cancer; ORR, objective response rate; Pts, patients; Pem, pemetrexed; Ref, reference; RT, radiotherapy; SCLC, small cell lung cancer; SD, stable disease; Tras, trastuzumab; Thi, thiotepa; TOPO, topotecan. 
TABLE 5 | Summary of landmark clinical trials of HER2-positive metastatic breast cancer.

\begin{tabular}{|c|c|c|c|c|c|c|c|c|}
\hline Study (NCT No.) & Phase & $\begin{array}{l}\text { Pts } \\
\text { (n) }\end{array}$ & Study design (n) & $\begin{array}{l}\text { Therapy } \\
\text { line }\end{array}$ & $\begin{array}{l}\text { CNSM } \\
\text { (n) }\end{array}$ & $\begin{array}{c}\text { BM } \\
\text { (n) }\end{array}$ & $\begin{array}{l}\text { LM } \\
\text { (n) }\end{array}$ & Survival (months) \\
\hline $\begin{array}{l}\text { EMILIA (86-88) } \\
\text { (NCT00829166) }\end{array}$ & III & 991 & T-DM1 (495) vs. Lap + Cap (496) & $2^{\text {nd }}$ & $+(90)$ & + & NA & $\begin{array}{l}\text { All: mPFS } 9.6 \text { vs. } 6.4(\mathrm{P}<0.001), \text { mOS } 29.9 \text { vs. } \\
25.9 \text { CNS: } \\
\text { CNSM: mPFS } 5.9 \text { vs. } 5.7(\mathrm{P}=0.9998), \text { mOS } \\
26.8 \text { vs. } 12.9(\mathrm{P}=0.0081)\end{array}$ \\
\hline $\begin{array}{l}\text { CLEOPATRA (89-91) } \\
\text { (NCT00567190) }\end{array}$ & III & 808 & $\begin{array}{l}\text { Per + Tras + Doc (402) vs. Pla + Tras } \\
+ \text { Doc (406) }\end{array}$ & $1^{\text {st }}$ & - & - & - & $\begin{array}{l}\text { All: } \mathrm{mPFS} 18.7 \text { vs. } 12.4 \text {, mOS } 57.1 \text { vs. } 40.8 \\
\text { CNSM: Median time to onset of CNSM } 15.0 \\
\text { vs. } 11.9(\mathrm{P}=0.0049) \\
\text { mOS } 34.4 \text { vs. } 26.3(\mathrm{P}=0.1139)\end{array}$ \\
\hline $\begin{array}{l}\text { LANDSCAPE (92) } \\
\text { (NCT00967031) }\end{array}$ & $\|$ & 45 & Lap + Cap & $1^{\text {st }}$ & + & $\begin{array}{c}+ \\
(45)\end{array}$ & NA & All/BM: mPFS 5.5, ORR 65.9\% \\
\hline $\begin{array}{l}\text { TH3RESA }(93,94) \\
\text { (NCT01419197) }\end{array}$ & III & 602 & $\begin{array}{l}\text { T-DM1(404) vs. Physician's Choice } \\
\text { (198) }\end{array}$ & $\begin{array}{l}\text { median } \\
\text { line: } 4^{\text {th }}\end{array}$ & + & $\begin{array}{c}+ \\
(72)\end{array}$ & NA & $\begin{array}{l}\text { All: mPFS } 6.2 \text { vs. } 3.3(P<0.0001) \text {, mOS } 22.7 \\
\text { vs. } 15.8 \text { ( } P=0.0007) \\
\text { BM: mPFS } 5.8 \text { vs. } 2.9 \text {, mOS } 17.3 \text { vs. } 12.6\end{array}$ \\
\hline $\begin{array}{l}\text { BOLERO-3 (95) } \\
\text { (NCT01007942) }\end{array}$ & III & 569 & $\begin{array}{l}\text { Eve+ Vin + Tras (284) vs. Pla + Vin + } \\
\text { Tras (285) }\end{array}$ & $2^{\text {nd }}$ & $+(27)$ & - & - & All: mPFS 7.00 vs. 5.78 (P=0.0067) \\
\hline $\begin{array}{l}\text { NEfERT-T (96) } \\
\text { (NCT00915018) }\end{array}$ & III & 479 & Ner + Pac (242) vs. Tras + Pac (237) & $1^{\text {st }}$ & $+(18)$ & + & NA & $\begin{array}{l}\text { All: } \mathrm{mPFS} 12.9 \text { vs. } 12.9(\mathrm{P}=0.89) \\
\text { Incidence of } \mathrm{CNS} \text { recurrences: } 8.3 \% \text { vs. } 17.3 \% \\
(\mathrm{P}=0.002)\end{array}$ \\
\hline $\begin{array}{l}\text { MARIANNE (97) } \\
\text { (NCT01120184) }\end{array}$ & III & 1095 & $\begin{array}{l}\text { Tras + Tax (365) vs. T-DM1 + Pla } \\
\text { (367) vs. T-DM1 + Per (363) }\end{array}$ & $1^{\text {st }}$ & NA & NA & NA & $\begin{array}{l}\text { All: mPFS } 13.7 \text { vs. } 12.9 \text { (P=0.31) vs. } 15.2 \\
(\mathrm{P}=0.14)\end{array}$ \\
\hline $\begin{array}{l}\text { ALTERNATIVE (98) } \\
\text { (NCT01160211) }\end{array}$ & III & 355 & $\begin{array}{l}\text { Lap + Tras + Al (120) vs. Tras + Al } \\
(117) \text { vs. Lap + Al (118) }\end{array}$ & $1^{\text {st }} / 2^{\text {nd }}$ & - & - & - & $\begin{array}{l}\text { All: mPFS } 11 \text { vs. } 5.7(\mathrm{P}=0.0064) \text { vs. } 8.3 \\
(\mathrm{P}=0.0361) \\
\text { mOS } 46.0(\mathrm{P}=0.07) \text { vs. } 40.0 \text { vs. } 45.1(\mathrm{P}=0.440)\end{array}$ \\
\hline $\begin{array}{l}\text { PHENIX (99) } \\
\text { (NCT02973737) }\end{array}$ & III & 279 & Pyr + Cap (185) vs. Pla + Cap (94) & $2^{\text {nd }}$ & + & $\begin{array}{c}+ \\
(31)\end{array}$ & NA & $\begin{array}{l}\text { All: } \mathrm{mPFS} 11.1 \text { vs. } 4.1(\mathrm{P}<0.001) \\
\text { BM: mPFS } 4.2 \text { vs. } 6.9(\mathrm{P}=0.011)\end{array}$ \\
\hline $\begin{array}{l}\text { PERUSE (100) } \\
\text { (NCT01572038) }\end{array}$ & III & 1436 & $\begin{array}{l}\text { Per + Tras + Tax (Doc 775, Pac 589, } \\
\text { Nab-Pac 65) }\end{array}$ & $1^{\text {st }}$ & + & + & NA & $\begin{array}{l}\text { All: mPFS } 20.6 \text { (Doc 19.6, Pac 23.0, Nab-Pac } \\
\text { 18.1) }\end{array}$ \\
\hline $\begin{array}{l}\text { TBCRC } 022(101) \\
\text { (NCT01494662) }\end{array}$ & $\|$ & 49 & $\begin{array}{l}\text { Ner + Cap (No prior Lap: 37, Prior } \\
\text { Lap: 12) }\end{array}$ & Any & + & $\begin{array}{c}+ \\
(49)\end{array}$ & $\begin{array}{c}+ \\
(3)\end{array}$ & $\begin{array}{l}\text { ALL/BM: No prior Lap: mPFS 5.5, mOS 13.3, } \\
\text { Prior Lap: mPFS 3.1, mOS } 15.1 \\
\text { LM:3 patients had PD, PR, SD, respectively }\end{array}$ \\
\hline $\begin{array}{l}\text { KAMILLA }(102,103) \\
\text { (NCT01702571) }\end{array}$ & III & 2003 & $\mathrm{~T}-\mathrm{DM} 1$ & Any & + & $\begin{array}{c}+ \\
(398)\end{array}$ & NA & $\begin{array}{l}\text { Cohort1: mPFS 6.9, mOS } 27.2 \\
\text { BM: mPFS 5.5, mOS } 18.9\end{array}$ \\
\hline NALA (104) (NCT01808573) & III & 621 & Ner + Cap (307) vs. Lap + Cap (314) & $\geq 3^{\text {rd }}$ & + & $\begin{array}{c}+ \\
(101)\end{array}$ & - & $\begin{array}{l}\text { All: Mean PFS: } 8.8 \text { vs. } 6.6(P=0.0003) \\
\text { BM: mPFS 5.5, mOS } 18.9\end{array}$ \\
\hline $\begin{array}{l}\text { PUFFIN (105) } \\
\text { (NCT00567190) }\end{array}$ & III & 243 & $\begin{array}{l}\text { Per + Tras + Doc (122) vs. Pla + Tras } \\
+ \text { Doc (121) }\end{array}$ & $1^{\text {st }}$ & - & - & - & All: mPFS 14.5 vs. 12.4 , mOS 57.1 vs. 40.8 \\
\hline $\begin{array}{l}\text { DESTINY-Breast } 01 \text { (106, } \\
\text { 107) (NCT03248492) }\end{array}$ & $\|$ & 184 & DS-8201 & $\begin{array}{l}\text { median } \\
\text { line: } 6^{\text {th }}\end{array}$ & + & $\begin{array}{c}+ \\
(24)\end{array}$ & NA & $\begin{array}{l}\text { All: } \mathrm{mPFS} 19.4, \text { mOS } 24.6 \\
\text { BM: mPFS } 18.1\end{array}$ \\
\hline $\begin{array}{l}\text { HER2CLIMB (108) } \\
\text { (NCT02614794) }\end{array}$ & $\|$ & 612 & $\begin{array}{l}\text { Tuc + Tras+ Cap (410) vs. Pla + Tras } \\
+ \text { Cap (202) }\end{array}$ & $\begin{array}{l}\text { median } \\
\text { line: } 3^{\text {rd }}\end{array}$ & + & $\begin{array}{c}+ \\
(219)\end{array}$ & - & $\begin{array}{l}\text { All: mPFS } 7.8 \text { vs. } 5.6(\mathrm{P}<0.001) \text {, mOS } 21.9 \text { vs. } \\
17.4(\mathrm{P}=0.005) \\
\text { BM: mPFS } 7.6 \text { vs. } 5.4(\mathrm{P}<0.001)\end{array}$ \\
\hline $\begin{array}{l}\text { PHOEBE (109) } \\
(\text { NCT03080805) }\end{array}$ & III & 266 & Pyr + Cap (134) vs. Lap + Cap (132) & $\leq 3$ & - & - & - & All: mPFS 12.5 vs. $6.8(\mathrm{P}<0.0001)$ \\
\hline $\begin{array}{l}\text { SOPHIA (110) } \\
(\text { NCT02492711) }\end{array}$ & III & 536 & $\begin{array}{l}\text { Mar + Chemo (266) vs. Tras + } \\
\text { Chemo (270) }\end{array}$ & $\geq 2$ & + & $\stackrel{+}{(71)}$ & NA & $\begin{array}{l}\text { All: mPFS } 5.8 \text { vs. } 4.9(P=0.03), \text { mOS } 21.6 \text { vs. } \\
19.8(P=0.33)\end{array}$ \\
\hline
\end{tabular}

Al, aromatase inhibitor; BM, brain metastases; Cap, capecitabine; CNS, central nervous system; CNSM, central nervous system metastases; Doc, Docetaxel; DS-8201, Trastuzumab deruxtecan; Eve, everolimus; Lap, lapatinib; LM, leptomeningeal metastases; Mar, Margetuximab; mOS, median overall survival; mPFS, median progression-free survival; NA, not available; Nab-Pac, nab-paclitaxel; Ner, neratinib; Pac, paclitaxel; PD, Progressive disease; Per, pertuzumab; Pla, placebo; Pts, patients; PR, partial response; Pyr, pyrotinib; Ref, reference; Tax, taxane; T-DM1, trastuzumab emtansine; Tuc, tucatinib; Tem, temozolomide; SD, stable disease; Vin, vinorelbine.

that the therapeutic outcomes were satisfactory in several case reports where intrathecal trastuzumab was used (Table 1). There was no observational study discussed solely for LM of HER2positive BC (Table 2), indicating that there is still a dearth of attention on LM of HER2-positive BC, which necessitates further efforts and exploration, also may be due to premature cases inclusion (during a time when the idea of HER2 was unknown) and the difficulty in case collection. Previous landmark clinical trials for patients with HER2-positive MBC either excluded patients with LM or did not specify whether patients with LM were included (Table 5). And the number of patients with LM was too small in TBCRC 022 study to draw firm conclusion. However, the CLEOPATRA study has shown that pertuzumab + trastuzumab + docetaxel delayed the occurrence of BM, and patients with BM tend to have a better survival in this group. Gratifyingly, the DESTINY-Breast 01, EMILIA, LANDSCAPE, HER2CLIMB, and NALA study respectively confirmed that DS8201, T-DM1, lapatinib, tucatinib, and neratinib are effective in HER2-positive BC with CNS metastasis. And a recent phase II clinical trial enrolled thirteen patients (tumor agnostic) with LM 
including BC showed a benefit of pembrolizumab, which suggests that immunotherapy also has a potential in the treatment for LM (Table 4). Unfortunately, T-DM1, DS-8201, pertuzumab, tucatinib, neratinib, and pembrolizumab were not yet available in mainland China at the time our patient was diagnosed as LM. Moreover, patients with LM are generally in poor condition, making high-intensity treatment regimens difficult to accept. And intrathecal trastuzumab is an off-label medication used in China. Hence, newer, better, safer, and more effective treatment options for HER2-positive BC with LM are urgently needed.

Pyrotinib is a novel irreversible epidermal growth factor receptor (EGFR)/HER2 dual TKI. Currently, several clinical trials, such as the PHOEBE study (NCT03080805) and the PHENIX study (NCT02973737) which were presented orally by Chinese scholars at the ASCO meeting, have confirmed that pyrotinib is effective and tolerable in HER2-positive MBC. The ORR (67.2\% vs. 51.5\%) and mPFS (12.5 vs. 6.9 months, $p<.0001)$ in the capecitabine plus pyrotinib group were superior to lapatinib plus capecitabine group in patients with HER2positive $\mathrm{MBC}$, who had been given taxanes, anthracycline, and/ or trastuzumab and recruited by PHOEBE study. Compared with the combination of placebo and capecitabine, pyrotinib plus capecitabine was demonstrated to significantly prolong the mPFS (11.1 vs. 4.1 months, $p<.001$ ) in patients with HER2positive MBC previously treated with trastuzumab and taxanes in the PHENIX study. The subgroup analysis indicated that pyrotinib plus capecitabine prolonged $\mathrm{mPFS}$ in patients with $\mathrm{BM}$ at baseline by 2.7 months compared with the placebo group (6.9 vs. 4.2 months, $p=.011)$ with fewer BM progression $(73.3 \%$ vs. $87.5 \%$ ), and longer median time to BM progression (168 vs. 127 days). Among patients without BM, the pyrotinib group had a lower incidence of BM than the placebo group (1.2\% vs. $3.6 \%)$, and a longer median time to developing new BM (397.5 vs. 132.0 days). Moreover, preliminary results from an ongoing study (NCT03691051) in the Chinese population presented at the 2020 ESMO meeting showed that combining pyrotinib with capecitabine resulted in an ORR of $76.9 \%$ in HER2-positive MBC with BM (112). In terms of mechanism, the molecular weight of pyrotinib is $815.22 \mathrm{Da}(<1000 \mathrm{Da})$, which could allow pyrotinib to penetrate past the cell membrane and operate directly on intracellular targets. Hence, the effectiveness in CNS metastases may be due to the possibility that pyrotinib may penetrate the blood-brain barrier as a small-molecule antiHER2 TKI like lapatinib (113). And different from lapatinib, pyrotinib can irreversibly and simultaneously inhibit HER1, HER2, and HER4, thereby blocking RAS/RAF/MEK/MAPK and PI3K/AKT signaling pathways and inhibiting the proliferation of tumor cells $(114,115)$. Here, by this case, we have proven that pyrotinib is efficient in HER2-positive LM, although its efficiency might have partly owed to its combinational application with other drugs. But poor compliance of the patient with intrathecal methotrexate led to irregular and insufficient intrathecal administration and discontinuation of methotrexate before disease progression. In addition, leukodystrophy was also tracked in MRI. Therefore, intrathecal methotrexate may play a limited role in the treatment of the patient.

Furthermore, metronomic chemotherapy was a feasible treatment of advanced cancer, particularly those in poor physical conditions, such as the elderly (116). The effectiveness and safety of oral vinorelbine metronomic chemotherapy in treating elderly $\mathrm{MBC}$ patients have since been established by a phase II clinical investigation which found a mPFS of 7.7 months and a mOS of 15.9 months in the entire population of patients, comparable to traditional chemotherapy findings. There was no drug reduction or withdrawal and no grade 3-4 adverse events occurred. Besides, the VICTOR-2 study demonstrated that the metronomic vinorelbine plus capecitabine was curative for $\mathrm{MBC}$ with a low incidence of grade 3-4 adverse events and no incidents impairing patients' quality of life (117). For that reason, in the beginning, when our patient could not tolerate traditional chemotherapy, we advised the patient to try metronomic chemotherapy. Also, a study by Wildiers $\mathrm{H}$ et al. showed that there was a difference of 7-months in the mPFS between the two evaluated groups (12.7 months for trastuzumab and pertuzumab plus metronomic oral cyclophosphamide vs. 5.6 months for trastuzumab plus pertuzumab, $p=.12$ ), highlighting the advantages of combining metronomic chemotherapy with targeted therapy to treat HER2-positive MBC and giving credibility to our choice of treatment (118).

There are several limitations of the present study that should be addressed. Firstly, the literature review for LM in HER2positive $\mathrm{BC}$ found little findings in observational studies and clinical trials, focusing instead on case reports or case series, which were truly subject to the current state of research. Secondly, the literature research was performed by "keywords", which may lead to inevitable omissions and it was difficult to describe all information for each study. Thirdly, given the present complete-matching literature, we were unable to draw a firm conclusion for optimal treatment regimen in HER2positive BC with LM from nonrandomized controlled studies or case report carefully selected by clinicians with selection bias.

Encouragingly, the patient responded significantly with the combination of pyrotinib-targeted therapy and metronomic vinorelbine, with a 16- month survival time and enhanced quality of life. As far as we know, the survival time of the patient has exceeded the current average level, as reported in the past literature (119). Besides, this tailored treatment regimen was quite well-tolerated, and efficiently relieved symptoms of the patient caused by leptomeningeal disease, which exceeded our expectations.

\section{CONCLUSION}

The treatment for HER2-positive BC with LM is still at the exploratory phase, though, with no revolutionary breakthrough in recent years. Clinical trials focused on many novel drugs such as T-DM1, DS8201, tucatinib, and even immunotherapy have been conducted extensively. This case is merely a one-person report but revealed that pyrotinib plus metronomic vinorelbine was effective and tolerable in HER2-positive BC with LM. Based 
on multidisciplinary cooperation to develop the final treatment regimen, we constituted a successful step forward. Therefore, the treatment strategy of pyrotinib with metronomic vinorelbine is worthy to be further investigated in large-scale clinical trials for HER2-positive BC patients with LM to improve the prognosis of this population.

\section{AUTHOR CONTRIBUTIONS}

Conceptualization: YC and HL. Treatment decision-making and discussions: MS, LX, HG, RT, LS, BZ, BC, and HL. Data collection and analysis: YC and SY. Manuscript writing: YC. Final approval of manuscript: HL. All authors contributed to the article and approved the submitted version.

\section{REFERENCES}

1. Bray F, Ferlay J, Soerjomataram I, Siegel RL, Torre LA, Jemal A. Global Cancer Statistics 2018: GLOBOCAN Estimates of Incidence and Mortality Worldwide for 36 Cancers in 185 Countries. CA: Cancer J Clin (2018) 68:394-424. doi: 10.3322/caac.21492

2. Chamberlain MC. Neoplastic Meningitis. Neurol (2006) 12:179-87. doi: 10.1097/01.nrl.0000219732.33321.cc

3. Wang R, Zhu Y, Liu X, Liao X, He J, Niu L. The Clinicopathological Features and Survival Outcomes of Patients With Different Metastatic Sites in Stage IV Breast Cancer. BMC Cancer (2019) 19:1091. doi: 10.1186/s12885-0196311-z

4. Chen MT, Sun HF, Zhao Y, Fu WY, Yang LP, Gao SP, et al. Comparison of Patterns and Prognosis Among Distant Metastatic Breast Cancer Patients by Age Groups: A SEER Population-Based Analysis. Sci Rep (2017) 7:9254. doi: 10.1038/s41598-017-10166-8

5. Bowman KM, Kumthekar P. Medical Management of Brain Metastases and Leptomeningeal Disease in Patients With Breast Carcinoma. Future Oncol (London England) (2018) 14:391-407. doi: 10.2217/fon-2017-0255

6. Nakao T, Okuda T, Fujita M. \& Kato, A. A Case of Leptomeningeal Metastases of Human Epidermal Growth Factor Receptor 2-Positive Breast Cancer That Responded Well to Lapatinib Plus Capecitabine. Surg Neurol Int (2019) 10:131. doi: 10.25259/sni-106-2019

7. Sacco K, Muhammad A, Saleem W, Alshaker H, Monzon L, Islam MR, et al. Leptomeningeal Carcinomatosis as the Primary Presentation of Relapse in Breast Cancer. Oncol Lett (2016) 12:779-82. doi: 10.3892/ol.2016.4745

8. Niwińska A, Rudnicka H, Murawska M. Breast Cancer Leptomeningeal Metastasis: Propensity of Breast Cancer Subtypes for Leptomeninges and the Analysis of Factors Influencing Survival. Med Oncol (Northwood London England) (2013) 30:408. doi: 10.1007/s12032-012-0408-4

9. de Azevedo CR, Cruz MR, Chinen LT, Peres SV, Peterlevitz MA, de Azevedo Pereira AE, et al. Meningeal Carcinomatosis in Breast Cancer: Prognostic Factors and Outcome. J Neuro-oncol (2011) 104:565-72. doi: 10.1007/ s11060-010-0524-y

10. Morikawa A, Jordan L, Rozner R, Patil S, Boire A, Pentsova E, et al. Characteristics and Outcomes of Patients With Breast Cancer With Leptomeningeal Metastasis. Clin Breast Cancer (2017) 17:23-8. doi: 10.1016/j.clbc.2016.07.002

11. Lee S, Ahn HK, Park YH, Nam DH, Lee JI, Park W, et al. Leptomeningeal Metastases From Breast Cancer: Intrinsic Subtypes may Affect Unique Clinical Manifestations. Breast Cancer Res Treat (2011) 129:809-17. doi: 10.1007/s10549-011-1682-0

12. Abouharb S, Ensor J, Loghin ME, Katz R, Moulder SL, Esteva FJ, et al. Leptomeningeal Disease and Breast Cancer: The Importance of Tumor Subtype. Breast Cancer Res Treat (2014) 146:477-86. doi: 10.1007/s10549014-3054-z

13. García FJV, Carrión NP, de la Cruz-Merino L. Long-Term Complete Response to Intrathecal Trastuzumab in a Patient With Leptomeningeal

\section{FUNDING}

We also would like to acknowledge the funding support of the National Natural Science Foundation of China (Grant No. 81902713), the Breast Disease Research Foundation of Shandong Provincial Medical Association (Grant No. YXH2020ZX066) and the Chinese Society of Clinical Oncology-Heng Rui Cancer Research Foundation (Grant No. Y-HR2019-0432; Y-HR2018-121).

\section{ACKNOWLEDGMENTS}

We sincerely thank the patient for her willingness to share all her experiences and feelings with the public.

Carcinomatosis Due to Her2- Overexpressing Breast Cancer: Case Report. Medicine (2020) 99:e18298. doi: 10.1097/md.0000000000018298

14. Ricciardi GRR, Russo A, Franchina T, Schifano S, Mastroeni G, Santacaterina A, et al. Efficacy of T-DM1 for Leptomeningeal and Brain Metastases in a HER2 Positive Metastatic Breast Cancer Patient: New Directions for Systemic Therapy - a Case Report and Literature Review. BMC Cancer (2018) 18:97. doi: 10.1186/s12885-018-3994-5

15. Ji G, Zhang CY, Guan S, Yao XY. Erlotinib for Progressive Brain and Leptomeningeal Metastases From HER2-Positive Breast Cancer After Treatment Failure With Trastuzumab and Lapatinib: Experience and Review of Literature. Clin Breast Cancer (2018) 18(5):e759-65. doi: 10.1016/j.clbc.2018.07.022

16. Kordbacheh T, Law WY, Smith IE. Sanctuary Site Leptomeningeal Metastases in HER-2 Positive Breast Cancer: A Review in the Era of Trastuzumab. Breast (2016) 26:54-8. doi: 10.1016/j.breast.2015.11.005

17. Park WY, Kim HJ, Kim K, Bae SB, Lee SC. Intrathecal Trastuzumab Treatment in Patients With Breast Cancer and Leptomeningeal Carcinomatosis. Cancer Res Treat Off J Korean Cancer Assoc (2015) 48:843-7. doi: 10.4143/crt.2014.234

18. Vincent A, Lesser G, Brown D, Vern-Gross T, Metheny-Barlow L, Lawrence J, et al. Prolonged Regression of Metastatic Leptomeningeal Breast Cancer That has Failed Conventional Therapy: A Case Report and Review of the Literature. J Breast Cancer (2013) 16:122-6. doi: 10.4048/jbc.2013.16.1.122

19. Martens J, Venuturumilli P, Corbets L, Bestul D. Rapid Clinical and Radiographic Improvement After Intrathecal Trastuzumab and Methotrexate in a Patient With HER-2 Positive Leptomeningeal Metastases. Acta Oncol (Stockholm Sweden) (2013) 52:175-8. doi: 10.3109/0284186x.2012.689857

20. Hofer S, Mengele K, Stemmler H, Schmitt M, Pestalozzi B. Intrathecal Trastuzumab: Dose Matters. Acta Oncol (Stockholm Sweden) (2012) 51:9556. doi: 10.3109/0284186x.2012.673736

21. Mego M, Sycova-Mila Z, Obertova J, Rajec J, Liskova S, Palacka P, et al. Intrathecal Administration of Trastuzumab With Cytarabine and Methotrexate in Breast Cancer Patients With Leptomeningeal Carcinomatosis. Breast (2011) 20:478-80. doi: 10.1016/j.breast.2011.05.007

22. Oliveira M, Braga S, Passos-Coelho JL, Fonseca R, Oliveira J. Complete Response in HER2+ Leptomeningeal Carcinomatosis From Breast Cancer With Intrathecal Trastuzumab. Breast Cancer Res Treat (2011) 127:841-4. doi: 10.1007/s10549-011-1417-2

23. Ferrario C, Davidson A, Bouganim N, Aloyz R, Panasci LC. Intrathecal Trastuzumab and Thiotepa for Leptomeningeal Spread of Breast Cancer. Ann Oncol: Off J Eur Soc Med Oncol (2009) 20:792-5. doi: 10.1093/annonc/mdp019

24. Hoffmann AL, Buhk JH, Strik H. Neoplastic Meningitis From Breast Cancer: Feasibility and Activity of Long-Term Intrathecal Liposomal Ara-C Combined With Dose-Dense Temozolomide. Anticancer Res (2009) 29:5191-5.

25. Shigekawa T, Takeuchi H, Misumi M, Matsuura K, Sano H, Fujiuchi N, et al. Successful Treatment of Leptomeningeal Metastases From Breast Cancer Using the Combination of Trastuzumab and Capecitabine: A Case Report. Breast Cancer (Tokyo Japan) (2009) 16:88-92. doi: 10.1007/s12282-008-0056-x 
26. Mir O, Ropert S, Alexandre J, Lemare F, Goldwasser F. High-Dose Intrathecal Trastuzumab for Leptomeningeal Metastases Secondary to HER-2 Overexpressing Breast Cancer. Ann Oncol: Off J Eur Soc Med Oncol (2008) 19:1978-80. doi: 10.1093/annonc/mdn654

27. Stemmler HJ, Mengele K, Schmitt M, Harbeck N, Laessig D, Herrmann KA, et al. Intrathecal Trastuzumab (Herceptin) and Methotrexate for Meningeal Carcinomatosis in HER2-Overexpressing Metastatic Breast Cancer: A Case Report. Anti-cancer Drugs (2008) 19:832-6. doi: 10.1097/CAD. ob013e32830b58b0

28. Ekenel M, Hormigo AM, Peak S, Deangelis LM, Abrey LE. Capecitabine Therapy of Central Nervous System Metastases From Breast Cancer. J Neuro-oncol (2007) 85:223-7. doi: 10.1007/s11060-007-9409-0

29. Platini C, Long J, Walter S. Meningeal Carcinomatosis From Breast Cancer Treated With Intrathecal Trastuzumab. Lancet Oncol (2006) 7:778-80. doi: 10.1016/s1470-2045(06)70864-6

30. Stemmler HJ, Schmitt M, Harbeck N, Willems A, Bernhard H, Lässig D, et al. Application of Intrathecal Trastuzumab (Herceptintrade Mark) for Treatment of Meningeal Carcinomatosis in HER2-Overexpressing Metastatic Breast Cancer. Oncol Rep (2006) 15:1373-7. doi: 10.3892/ or.15.5.1373

31. Hikino H, Yamada T, Johbara K, Obayashi N, Ozaki N. Potential Role of Chemo-Radiation With Oral Capecitabine in a Breast Cancer Patient With Central Nervous System Relapse. Breast (2006) 15:97-9. doi: 10.1016/ j.breast.2005.03.006

32. Ozdogan M, Samur M, Bozcuk HS, Sagtas E, Yildiz M, Artac M, et al. Durable Remission of Leptomeningeal Metastasis of Breast Cancer With Letrozole: A Case Report and Implications of Biomarkers on Treatment Selection. Jpn J Clin Oncol (2003) 33:229-31. doi: 10.1093/jjco/hyg046

33. Okada Y, Abe T, Shinozaki M, Tanaka A, Kobayashi M, Hiromichi G, et al. Evaluation of Imaging Findings and Prognostic Factors After Whole-Brain Radiotherapy for Carcinomatous Meningitis From Breast Cancer: A Retrospective Analysis. Medicine (2020) 99:e21333. doi: 10.1097/ md.0000000000021333

34. Chahal J, Stopeck A, Clarke K, Livingston R, Chalasani P. Intravenous Thiotepa for Treatment of Breast Cancer-Related Leptomeningeal Carcinomatosis: Case Series. Neurol Sci: Off J Ital Neurol Soc Ital Soc Clin Neurophysiol (2015) 36:1691-3. doi: 10.1007/s10072-015-2259-1

35. Du C, Hong R, Shi Y, Yu X, Wang J. Leptomeningeal Metastasis From Solid Tumors: A Single Center Experience in Chinese Patients. J Neuro-oncol (2013) 115:285-91. doi: 10.1007/s11060-013-1228-x

36. Kim H, Lee E. A Retrospective Analysis of the Clinical Outcomes of Leptomeningeal Metastasis in Patients With Solid Tumors. Brain Tumor Res Treat (2018) 6:54-9. doi: 10.14791/btrt.2018.6.e12

37. Fusco J, Castañón E, Carranza OE, Zubiri L, Martín P, Espinós J, et al. Neurological and Cytological Response as Potential Early Predictors of Time-to-Progression and Overall Survival in Patients With Leptomeningeal Carcinomatosis Treated With Intrathecal Liposomal Cytarabine: A Retrospective Cohort Study. J Neuro-oncol (2013) 115:42935. doi: 10.1007/s11060-013-1241-0

38. Le Rhun E, Taillibert S, Zairi F, Kotecki N, Devos P, Mailliez A, et al. A Retrospective Case Series of 103 Consecutive Patients With Leptomeningeal Metastasis and Breast Cancer. J Neuro-oncol (2013) 113:83-92. doi: 10.1007/ s11060-013-1092-8

39. Jaeckle K, Dixon JG, Anderson SK, Moreno-Aspitia A, Colon-Otero G, Hebenstreit K, et al. Intra-CSF Topotecan in Treatment of Breast Cancer Patients With Leptomeningeal Metastases. Cancer Med (2020) 9:7935-42. doi: $10.1002 / \mathrm{cam} 4.3422$

40. Kingston B, Kayhanian H, Brooks C, Cox N, Chaabouni N, Redana S, et al. Treatment and Prognosis of Leptomeningeal Disease Secondary to Metastatic Breast Cancer: A Single-Centre Experience. Breast (Edinburgh Scotland) (2017) 36:54-9. doi: 10.1016/j.breast.2017.07.015

41. Cochereau D, Da Costa S, Le Maignan C, Gauthier H, Cochereau J, Espié M, et al. [Intrathecal Methotrexate in Breast Cancer Meningeal Carcinomatosis - Experience With a New Administration Schedule]. Bull Cancer (2016) 103:444-54. doi: 10.1016/j.bulcan.2016.02.002

42. Quigley M, Fukui O, Chew B, Bhatia S, Karlovits S. The Shifting Landscape of Metastatic Breast Cancer to the CNS. Neurosurg Rev (2013) 36:377-82. doi: 10.1007/s10143-012-0446-6
43. Lara-Medina F, Crismatt A, Villarreal-Garza C, Alvarado-Miranda A, FloresHernández L, González-Pinedo M, et al. Clinical Features and Prognostic Factors in Patients With Carcinomatous Meningitis Secondary to Breast Cancer. Breast J (2012) 18:233-41. doi: 10.1111/j.1524-4741.2012.01228.x

44. Griguolo G, Pouderoux S, Dieci MV, Jacot W, Bourgier C, Miglietta F, et al. Clinicopathological and Treatment-Associated Prognostic Factors in Patients With Breast Cancer Leptomeningeal Metastases in Relation to Tumor Biology. Oncol (2018) 23:1289-99. doi: 10.1634/theoncologist.2018-0200

45. Comte A, Jdid W, Guilhaume MN, Kriegel I, Piperno-Neumann S, Dieras V, et al. Survival of Breast Cancer Patients With Meningeal Carcinomatosis Treated by Intrathecal Thiotepa. J Neuro-oncol (2013) 115:445-52. doi: 10.1007/s11060-013-1244-x

46. Gauthier H, Guilhaume MN, Bidard FC, Pierga JY, Girre V, Cottu PH, et al. Survival of Breast Cancer Patients With Meningeal Carcinomatosis. Ann Oncol: Off J Eur Soc Med Oncol (2010) 21:2183-7. doi: 10.1093/annonc/ mdq232

47. Rudnicka H, Niwińska A, Murawska M. Breast Cancer Leptomeningeal Metastasis-the Role of Multimodality Treatment. J Neuro-oncol (2007) 84:57-62. doi: 10.1007/s11060-007-9340-4

48. Niwińska A, Pogoda K, Michalski W, Kunkiel M, Jagiełło-Gruszfeld A. Determinants of Prolonged Survival for Breast Cancer Patient Groups With Leptomeningeal Metastasis (LM). J Neuro-oncol (2018) 138:191-8. doi: 10.1007/s11060-018-2790-z

49. Niwińska A, Rudnicka H, Murawska M. Breast Cancer Leptomeningeal Metastasis: The Results of Combined Treatment and the Comparison of Methotrexate and Liposomal Cytarabine as Intra-Cerebrospinal Fluid Chemotherapy. Clin Breast Cancer (2015) 15:66-72. doi: 10.1016/ j.clbc.2014.07.004

50. Clatot F, Philippin-Lauridant G, Ouvrier MJ, Nakry T, Laberge-Le-Couteulx S, Guillemet C, et al. Clinical Improvement and Survival in Breast Cancer Leptomeningeal Metastasis Correlate With the Cytologic Response to Intrathecal Chemotherapy. J Neuro-oncol (2009) 95:421-6. doi: 10.1007/ s11060-009-9940-2

51. Kosmas C, Malamos NA, Tsavaris NB, Stamataki M, Gregoriou A, Rokana S, et al. Isolated Leptomeningeal Carcinomatosis (Carcinomatous Meningitis) After Taxane-Induced Major Remission in Patients With Advanced Breast Cancer. Oncology (2002) 63:6-15. doi: 10.1159/000065714

52. Yust-Katz S, Garciarena P, Liu D, Yuan Y, Ibrahim N, Yerushalmi R, et al. Breast Cancer and Leptomeningeal Disease (LMD): Hormone Receptor Status Influences Time to Development of LMD and Survival From LMD Diagnosis. J Neuro-oncol (2013) 114:229-35. doi: 10.1007/s11060-013-1175-6

53. Chamberlain M, Johnston S, Glantz M. Neoplastic Meningitis-Related Prognostic Significance of the Karnofsky Performance Status. Arch Neurol (2009) 66:74-8. doi: 10.1001/archneurol.2008.506

54. Yu H, Mitsumori M, Nagata Y, Katakura Y, Kokubo M, Oya N, et al. Meningeal Carcinomatosis in Patients With Breast Cancer: Report of 8 Patients. Breast Cancer (Tokyo Japan) (2001) 8:74-8. doi: 10.1007/ bf02967482

55. Chamberlain M, Kormanik P. Carcinomatous Meningitis Secondary to Breast Cancer: Predictors of Response to Combined Modality Therapy. J Neuro-oncol (1997) 35:55-64. doi: 10.1023/a:1005803918194

56. Jayson G, Howell A, Harris M, Morgenstern G, Chang J, Ryder WD. Carcinomatous Meningitis in Patients With Breast Cancer. An Aggressive Disease Variant. Cancer (1994) 74:3135-41. doi: 10.1002/1097-0142 (19941215)74:12<3135::aid-cncr2820741212>3.0.co;2-8

57. Strady C, Ricciarelli A, Nasca S, Liautaud-Roger F, Coninx P. Carcinomatous Meningitis and Solid Tumours. Oncol Rep (2000) 7:203-7. doi: 10.3892/or.7.1.203

58. U.S. National Institutes of Health. Tucatinib, Trastuzumab, and Capecitabine for the Treatment of HER2+ LMD. Available at: https://www.clinicaltrials.gov/ ct2/show/NCT03501979 (Accessed January 11, 2021).

59. U.S. National Institutes of Health. Radiation Therapy Followed by Intrathecal Trastuzumab/Pertuzumab in HER2+ Breast Leptomeningeal Disease. Available at: https://www.clinicaltrials.gov/ct2/show/ NCT04588545 (Accessed January 11, 2021).

60. U.S. National Institutes of Health. DS-8201a for Treatment of $a B c$, BRain Mets, And Her2[+] Disease (DEBBRAH). Available at: https:// www.clinicaltrials.gov/ct2/show/NCT04420598 (Accessed January 11, 2021). 
61. U.S. National Institutes of Health. HER2-CAR T Cells in Treating Patients With Recurrent Brain or Leptomeningeal Metastases. Available at: https:// www.clinicaltrials.gov/ct2/show/NCT03696030 (Accessed January 11, 2021).

62. U.S. National Institutes of Health. Intermittent High-Dose Lapatinib in Tandem With Capecitabine for HER2 Overexpressed/Amplified Metastatic Breast Cancer With Central Nervous System (CNS) Metastases. Available at: https://www. clinicaltrials.gov/ct2/show/NCT02650752 (Accessed January 11, 2021).

63. U.S. National Institutes of Health. ANG1005 in Leptomeningeal Disease From Breast Cancer (ANGLeD). Available at: https://clinicaltrials.gov/ct2/ show/NCT03613181 (Accessed January 11, 2021).

64. U.S. National Institutes of Health.BATs in Patients With Breast Cancer and Leptomeningeal Metastases. Available at: https:/clinicaltrials.gov/ct2/show/ NCT03661424 (Accessed January 11, 2021).

65. U.S. National Institutes of Health. Prospective Evaluation Of High-Dose Systemic Methotrexate In Patients With Breast Cancer And Leptomeningeal Metastasis. Available at: https://clinicaltrials.gov/ct2/show/NCT02422641 (Accessed January 11, 2021).

66. U.S. National Institutes of Health. Clinical and Pharmacological Study With 2B3-101 in Patients With Breast Cancer and Leptomeningeal Metastases. Available at: https://clinicaltrials.gov/ct2/show/NCT01818713 (Accessed Jaunary 11, 2021).

67. U.S. National Institutes of Health. Liposomal Cytarabine and High-Dose Methotrexate in Treating Patients With Central Nervous System Metastases From Breast Cancer. Available at: https://clinicaltrials.gov/ct2/show/ NCT00992602 (Accessed January 11, 2021).

68. Le Rhun E, Wallet J, Mailliez A, Le Deley MC, Rodrigues I, Boulanger T, et al. Intrathecal Liposomal Cytarabine Plus Systemic Therapy Versus Systemic Chemotherapy Alone for Newly Diagnosed Leptomeningeal Metastasis From Breast Cancer. Neuro-oncol (2020) 22:524-38. doi: 10.1093/neuonc/noz201

69. Naidoo J, Schreck KC, Hu C, Douville CB, Santa-Maria CA, Connolly RM, et al. Anti-PD-1 for Patients With Leptomeningeal Metastasis From Advanced Solid Tumors: Efficacy, Safety, and Biomarkers of Response. J Clin Oncol: Off J Am Soc Clin Oncol (2020) 38. doi: 10.1200/JCO.2020.38.15_suppl.e14506

70. Malani R, Fleisher M, Kumthekar P, Lin X, Omuro A, Groves MD, et al. Cerebrospinal Fluid Circulating Tumor Cells as a Quantifiable Measurement of Leptomeningeal Metastases in Patients With HER2 Positive Cancer. J Neuro-oncol (2020) 148:599-606. doi: 10.1007/s11060-020-03555-Z

71. Pan Z, Yang G, He H, Cui J, Li W, Yuan T, et al. Intrathecal Pemetrexed Combined With Involved-Field Radiotherapy as a First-Line Intra-CSF Therapy for Leptomeningeal Metastases From Solid Tumors: A Phase I/II Study. Ther Adv Med Oncol (2020) 12:1758835920937953. doi: 10.1177/ 1758835920937953

72. Bonneau C, Paintaud G, Trédan O, Dubot C, Desvignes C, Dieras V, et al. Phase I Feasibility Study for Intrathecal Administration of Trastuzumab in Patients With HER2 Positive Breast Carcinomatous Meningitis. Eur J Cancer (Oxford England: 1990) (2018) 95:75-84. doi: 10.1016/j.ejca.2018.02.032

73. Wu P, Lin CH, Kuo CH, Chen WW, Yeh DC, Liao HW, et al. A Pilot Study of Bevacizumab Combined With Etoposide and Cisplatin in Breast Cancer Patients With Leptomeningeal Carcinomatosis. BMC Cancer (2015) 15:299. doi: 10.1186/s12885-015-1290-1

74. Ursu R, Taillibert S, Banissi C, Vicaut E, Bailon O, Le Rhun E, et al. Immunotherapy With CpG-ODN in Neoplastic Meningitis: A Phase I Trial. Cancer Sci (2015) 106:1212-8. doi: 10.1111/cas.12724

75. Bernardi R, Bomgaars L, Fox E, Balis FM, Egorin MJ, Lagattuta TF, et al. Phase I Clinical Trial of Intrathecal Gemcitabine in Patients With Neoplastic Meningitis. Cancer Chemother Pharmacol (2008) 62:355-61. doi: 10.1007/ s00280-007-0601-x

76. Chamberlain M, Tsao-Wei D, Groshen S. Phase II Trial of Intracerebrospinal Fluid Etoposide in the Treatment of Neoplastic Meningitis. Cancer (2006) 106:2021-7. doi: 10.1002/cncr.21828

77. Boogerd W, van den Bent MJ, Koehler PJ, Heimans JJ, van der Sande JJ, Aaronson NK, et al. The Relevance of Intraventricular Chemotherapy for Leptomeningeal Metastasis in Breast Cancer: A Randomised Study. Eur J Cancer (Oxford England: 1990) (2004) 40:2726-33. doi: 10.1016/j.ejca.2004.08.012

78. Blaney S, Heideman R, Berg S, Adamson P, Gillespie A, Geyer JR, et al. Phase I Clinical Trial of Intrathecal Topotecan in Patients With Neoplastic Meningitis. J Clin Oncol: Off J Am Soc Clin Oncol (2003) 21:143-7. doi: $10.1200 /$ jco.2003.04.053
79. Orlando L, Curigliano G, Colleoni M, Fazio N, Nole F, Martinelli G, et al. Intrathecal Chemotherapy in Carcinomatous Meningitis From Breast Cancer. Anticancer Res (2002) 22:3057-9.

80. Chamberlain M. A Phase II Trial of Intra-Cerebrospinal Fluid Alpha Interferon in the Treatment of Neoplastic Meningitis. Cancer (2002) 94:2675-80. doi: 10.1002/cncr.10547

81. Jaeckle K, Batchelor T, O'Day SJ, Phuphanich S, New P, Lesser G, et al. An Open Label Trial of Sustained-Release Cytarabine (DepoCyt) for the Intrathecal Treatment of Solid Tumor Neoplastic Meningitis. J Neurooncol (2002) 57:231-9. doi: 10.1023/a:1015752331041

82. Jaeckle K, Phuphanich S, Bent MJ, Aiken R, Batchelor T, Campbell T, et al. Intrathecal Treatment of Neoplastic Meningitis Due to Breast Cancer With a Slow-Release Formulation of Cytarabine. Br J Cancer (2001) 84:157-63. doi: 10.1054/bjoc.2000.1574

83. Glantz M, Jaeckle KA, Chamberlain MC, Phuphanich S, Recht L, Swinnen LJ, et al. A Randomized Controlled Trial Comparing Intrathecal SustainedRelease Cytarabine (DepoCyt) to Intrathecal Methotrexate in Patients With Neoplastic Meningitis From Solid Tumors. Clin Cancer Res: Off J Am Assoc Cancer Res (1999) 5:3394-402.

84. Grossman S, Finkelstein DM, Ruckdeschel JC, Trump DL, Moynihan T, Ettinger DS. Randomized Prospective Comparison of Intraventricular Methotrexate and Thiotepa in Patients With Previously Untreated Neoplastic Meningitis. Eastern Cooperative Oncology Group. J Clin Oncol: Off J Am Soc Clin Oncol (1993) 11:561-9. doi: 10.1200/jco.1993.11.3.561

85. Hitchins R, Bell D, Woods R, Levi J. A Prospective Randomized Trial of Single-Agent Versus Combination Chemotherapy in Meningeal Carcinomatosis. J Clin Oncol: Off J Am Soc Clin Oncol (1987) 5:1655-62. doi: 10.1200/jco.1987.5.10.1655

86. Diéras V, Miles D, Verma S, Pegram M, Welslau M, Baselga J, et al. Trastuzumab Emtansine Versus Capecitabine Plus Lapatinib in Patients With Previously Treated HER2-Positive Advanced Breast Cancer (EMILIA): A Descriptive Analysis of Final Overall Survival Results From a Randomised, Open-Label, Phase 3 Trial. Lancet Oncol (2017) 18:732-42. doi: 10.1016/ s1470-2045(17)30312-1

87. Verma S, Miles D, Gianni L, Krop IE, Welslau M, Baselga J, et al. Trastuzumab Emtansine for HER2-Positive Advanced Breast Cancer. New Engl J Med (2012) 367:1783-91. doi: 10.1056/NEJMoa1209124

88. Krop I, Lin NU, Blackwell K, Guardino E, Huober J, Lu M, et al. Trastuzumab Emtansine (T-DM1) Versus Lapatinib Plus Capecitabine in Patients With HER2-Positive Metastatic Breast Cancer and Central Nervous System Metastases: A Retrospective, Exploratory Analysis in EMILIA. Ann Oncol: Off J Eur Soc Med Oncol (2015) 26:113-9. doi: 10.1093/annonc/mdu486

89. Swain S, Miles D, Kim SB, Im YH, Im SA, Semiglazov V, et al. Pertuzumab, Trastuzumab, and Docetaxel for HER2-Positive Metastatic Breast Cancer (CLEOPATRA): End-of-Study Results From a Double-Blind, Randomised, Placebo-Controlled, Phase 3 Study. Lancet Oncol (2020) 21:519-30. doi: 10.1016/s1470-2045(19)30863-0

90. Swain S, Kim SB, Cortés J, Ro J, Semiglazov V, Campone M, et al. Pertuzumab, Trastuzumab, and Docetaxel for HER2-Positive Metastatic Breast Cancer (CLEOPATRA Study): Overall Survival Results From a Randomised, Double-Blind, Placebo-Controlled, Phase 3 Study. Lancet Oncol (2013) 14:461-71. doi: 10.1016/s1470-2045(13)70130-x

91. Swain S, Baselga J, Miles D, Im YH, Quah C, Lee LF, et al. Incidence of Central Nervous System Metastases in Patients With HER2-Positive Metastatic Breast Cancer Treated With Pertuzumab, Trastuzumab, and Docetaxel: Results From the Randomized Phase III Study CLEOPATRA. Ann Oncol: Off J Eur Soc Med Oncol (2014) 25:1116-21. doi: 10.1093/ annonc/mdu133

92. Bachelot T, Romieu G, Campone M, Diéras V, Cropet C, Dalenc F, et al. Lapatinib Plus Capecitabine in Patients With Previously Untreated Brain Metastases From HER2-Positive Metastatic Breast Cancer (LANDSCAPE): A Single-Group Phase 2 Study. Lancet Oncol (2013) 14:64-71. doi: 10.1016/ s1470-2045(12)70432-1

93. Krop I, Kim SB, González-Martín A, LoRusso PM, Ferrero JM, Smitt M, et al. Trastuzumab Emtansine Versus Treatment of Physician's Choice for Pretreated HER2-Positive Advanced Breast Cancer (TH3RESA): A Randomised, Open-Label, Phase 3 Trial. Lancet Oncol (2014) 15:689-99. doi: $10.1016 /$ s1470-2045(14)70178-0 
94. Krop I, Kim SB, González-Martín A, LoRusso PM, Ferrero JM, Smitt M, et al. Trastuzumab Emtansine Versus Treatment of Physician's Choice in Patients With Previously Treated HER2-Positive Metastatic Breast Cancer (TH3RESA): Final Overall Survival Results From a Randomised Open-Label Phase 3 Trial. Lancet Oncol (2017) 18:743-54. doi: 10.1016/s1470-2045(17)30313-3

95. André F, O'Regan R, Ozguroglu M, Toi M, Xu B, Jerusalem G, et al. Everolimus for Women With Trastuzumab-Resistant, HER2-Positive, Advanced Breast Cancer (BOLERO-3): A Randomised, Double-Blind, Placebo-Controlled Phase 3 Trial. Lancet Oncol (2014) 15:580-91. doi: 10.1016/s1470-2045(14)70138-x

96. Awada A, Colomer R, Inoue K, Bondarenko I, Badwe RA, Demetriou G, et al. Neratinib Plus Paclitaxel vs Trastuzumab Plus Paclitaxel in Previously Untreated Metastatic ERBB2-Positive Breast Cancer: The NEfERT-T Randomized Clinical Trial. JAMA Oncol (2016) 2:1557-64. doi: 10.1001/ jamaoncol.2016.0237

97. Perez E, Barrios C, Eiermann W, Toi M, Im YH, Conte P, et al. Trastuzumab Emtansine With or Without Pertuzumab Versus Trastuzumab Plus Taxane for Human Epidermal Growth Factor Receptor 2-Positive, Advanced Breast Cancer: Primary Results From the Phase III MARIANNE Study. J Clin Oncol: Off J Am Soc Clin Oncol (2017) 35:141-8. doi: 10.1200/jco.2016.67.4887

98. Johnston S, Phase III. Randomized Study of Dual Human Epidermal Growth Factor Receptor 2 (HER2) Blockade With Lapatinib Plus Trastuzumab in Combination With an Aromatase Inhibitor in Postmenopausal Women With HER2-Positive, Hormone Receptor-Positive Metastatic Breast Cancer: ALTERNATIVE. J Clin Oncol: Off J Am Soc Clin Oncol (2018) 36:741-8. doi: 10.1200/jco.2017.74.7824

99. Jiang ZF, Yan M, Hu X, Zhang QY, Ouyang QC, Feng JF, et al. Pyrotinib Combined With Capecitabine in Women With HER2+ Metastatic Breast Cancer Previously Treated With Trastuzumab and Taxanes: A Randomized Phase III Study. J Clin Oncol: Off J Am Soc Clin Oncol (2019) 37. doi: 10.1200/JCO.2019.37.15_suppl.1001

100. Bachelot T, Ciruelos E, Schneeweiss A, Puglisi F, Peretz-Yablonski T, Bondarenko I, et al. Preliminary Safety and Efficacy of First-Line Pertuzumab Combined With Trastuzumab and Taxane Therapy for HER2-Positive Locally Recurrent or Metastatic Breast Cancer (PERUSE). Ann Oncol: Off J Eur Soc Med Oncol (2019) 30:766-73. doi: 10.1093/annonc/ mdz061

101. Freedman R, Gelman RS, Anders CK, Melisko ME, Parsons HA, Cropp AM, et al. TBCRC 022: A Phase II Trial of Neratinib and Capecitabine for Patients With Human Epidermal Growth Factor Receptor 2-Positive Breast Cancer and Brain Metastases. J Clin Oncol: Off J Am Soc Clin Oncol (2019) 37:10819. doi: $10.1200 /$ jco.18.01511

102. Montemurro F, Delaloge S, Barrios CH, Wuerstlein R, Anton A, Brain E, et al. Trastuzumab Emtansine (T-DM1) in Patients With HER2-Positive Metastatic Breast Cancer and Brain Metastases: Exploratory Final Analysis of Cohort 1 From KAMILLA, a Single-Arm Phase IIIb Clinical Trial. Ann Oncol: Off J Eur Soc Med Oncol (2020) 31:1350-8. doi: 10.1016/ j.annonc.2020.06.020

103. Montemurro F, Ellis P, Anton A, Wuerstlein R, Delaloge S, Bonneterre J, et al. Safety of Trastuzumab Emtansine (T-DM1) in Patients With HER2Positive Advanced Breast Cancer: Primary Results From the KAMILLA Study Cohort 1. Eur J Cancer (Oxford England: 1990) (2019) 109:92-102. doi: 10.1016/j.ejca.2018.12.022

104. Saura C, Oliveira M, Feng YH, Dai MS, Chen SW, Hurvitz SA, et al. Neratinib Plus Capecitabine Versus Lapatinib Plus Capecitabine in HER2Positive Metastatic Breast Cancer Previously Treated With $\geq 2$ HER2Directed Regimens: Phase III NALA Trial. J Clin Oncol: Off J Am Soc Clin Oncol (2020) 38:3138-49. doi: 10.1200/jco.20.00147

105. Xu B, Li W, Zhang Q, Shao Z, Li Q, Wang X, et al. Pertuzumab, Trastuzumab, and Docetaxel for Chinese Patients With Previously Untreated HER2-Positive Locally Recurrent or Metastatic Breast Cancer (PUFFIN): A Phase III, Randomized, Double-Blind, Placebo-Controlled Study. Breast Cancer Res Treat (2020) 182:689-97. doi: 10.1007/s10549-020-05728-w

106. Modi S, Saura C, Yamashita T, Park YH, Kim SB, Tamura K, et al. Trastuzumab Deruxtecan in Previously Treated HER2-Positive Breast Cancer. New Engl J Med (2020) 382:610-21. doi: 10.1056/NEJMoa1914510

107. Modi S, Saura C, Yamashita T, Park YH, Krop IE. Updated Results From DESTINY-Breast01, a Phase 2 Trial of Trastuzumab Deruxtecan (T-DXd) in
HER2 Positive Metastatic Breast Cancer. SABCS; Abstract Session PD3 - Spotlight Poster Discussion 3-06 (2020). doi: 10.1158/1538-7445.SABCS20-PD3-06

108. Murthy R, Murthy R, Loi S, Okines A, Paplomata E, Hamilton E, Hurvitz SA, et al. Tucatinib, Trastuzumab, and Capecitabine for HER2-Positive Metastatic Breast Cancer. New Engl J Med (2020) 382:597-609. doi: 10.1056/NEJMoa1914609

109. Xu B, Xu B, Yan M, Ma F, Hu X, Feng J, Ouyang Q, et al. Pyrotinib Plus Capecitabine Versus Lapatinib Plus Capecitabine for the Treatment of HER2-Positive Metastatic Breast Cancer (PHOEBE): A Multicentre, OpenLabel, Randomised, Controlled, Phase 3 Trial. Lancet Oncol (2021) 22:35160. doi: 10.1016/s1470-2045(20)30702-6

110. Rugo H, Im SA, Cardoso F, Cortés J, Curigliano G, Musolino A, et al. Efficacy of Margetuximab vs Trastuzumab in Patients With Pretreated ERBB2Positive Advanced Breast Cancer: A Phase 3 Randomized Clinical Trial. JAMA Oncol (2021) 7(4):573-84. doi: 10.1001/jamaoncol.2020.7932

111. Le Rhun E, Weller M, Brandsma D, Van den Bent M, de Azambuja E, Henriksson R, et al. EANO-ESMO Clinical Practice Guidelines for Diagnosis, Treatment and Follow-Up of Patients With Leptomeningeal Metastasis From Solid Tumours. Ann Oncol: Off J Eur Soc Med Oncol (2017) 28:iv84-99. doi: 10.1093/annonc/mdx221

112. Yan M, Ouyang Q, Sun T, Niu L, Yang J, Li L, et al. Pyrotinib and Capecitabine for HER2-positive Metastatic Breast Cancer Patients With Previously Untreated Brain Metastases: A Single-Group Multicenter Phase II Study. Ann Oncol (2020) 31(suppl_4):S348-95. doi: 10.1016/annonc/annonc268

113. Roy V, Perez E. Beyond Trastuzumab: Small Molecule Tyrosine Kinase Inhibitors in HER-2-Positive Breast Cancer. Oncol (2009) 14:1061-9. doi: 10.1634/theoncologist.2009-0142

114. Traxler P. Tyrosine Kinases as Targets in Cancer Therapy - Successes and Failures. Expert Opin Ther Targets (2003) 7:215-34. doi: 10.1517/14728222.7.2.215

115. Xuhong JC, Qi XW, Zhang Y, Jiang J. Mechanism, Safety and Efficacy of Three Tyrosine Kinase Inhibitors Lapatinib, Neratinib and Pyrotinib in HER2-Positive Breast Cancer. Am J Cancer Res (2019) 9:2103-19.

116. Borne E, Desmedt E, Duhamel A, Mirabel X, Dziwniel V, Maire C, et al. Oral Metronomic Cyclophosphamide in Elderly With Metastatic Melanoma. Investig New Drugs (2010) 28:684-9. doi: 10.1007/s10637-009-9298-5

117. Cazzaniga M, Cortesi L, Ferzi A, Scaltriti L, Cicchiello F, Ciccarese M, et al. Metronomic Chemotherapy With Oral Vinorelbine (mVNR) and Capecitabine (mCAPE) in Advanced HER2-Negative Breast Cancer Patients: Is it a Way to Optimize Disease Control? Final Results of the VICTOR-2 Study. Breast Cancer Res Treat (2016) 160:501-9. doi: 10.1007/ s10549-016-4009-3

118. Wildiers H, Tryfonidis K, Lago DL, Vuylsteke P, Curigliano G, Waters S, et al. Pertuzumab and Trastuzumab With or Without Metronomic Chemotherapy for Older Patients With HER2-Positive Metastatic Breast Cancer (EORTC 75111-10114): An Open-Label, Randomised, Phase 2 Trial From the Elderly Task Force/Breast Cancer Group. Lancet Oncol. (2018) 19(3):323-36. doi: 10.1016/S1470-2045(18)30083-4

119. Franzoi MA, Hortobagyi GN. Leptomeningeal Carcinomatosis in Patients With Breast Cancer. Crit Rev Oncol Hematol (2019) 135:85-94. doi: 10.1016/ j.critrevonc. 2019.01 .020

Conflict of Interest: The authors declare that the research was conducted in the absence of any commercial or financial relationships that could be construed as a potential conflict of interest.

Publisher's Note: All claims expressed in this article are solely those of the authors and do not necessarily represent those of their affiliated organizations, or those of the publisher, the editors and the reviewers. Any product that may be evaluated in this article, or claim that may be made by its manufacturer, is not guaranteed or endorsed by the publisher.

Copyright (c) 2022 Chi, Shang, Xu, Gong, Tao, Song, Zhang, Yin, Cong and Li. This is an open-access article distributed under the terms of the Creative Commons Attribution License (CC BY). The use, distribution or reproduction in other forums is permitted, provided the original author(s) and the copyright owner(s) are credited and that the original publication in this journal is cited, in accordance with accepted academic practice. No use, distribution or reproduction is permitted which does not comply with these terms. 